\title{
Comparison of Risk Factors and Molecular Analysis of Right-Sided Colon and Left Sided Colon Cancer
}

\author{
Mauro Luisetto ${ }^{*}$, Behzad Nili Ahmadabadi ${ }^{2}$, Hossein Nili-Ahmadabadi ${ }^{3}$, Ahmed Yesvi Rafa ${ }^{4}$, Gamal Abdul $^{2}$ \\ Hamid $^{5}$, Ghulam Rasool Mashori ${ }^{6}$, luca cabianca ${ }^{7}$ and Oleg Yurievich Latyshev ${ }^{8}$ \\ ${ }^{1}$ European Specialist Lab medicine, Branch General Toxicology-pharmacy and pharmacology IMA academy, Italy \\ ${ }^{2}$ Innovative Pharmaceutical product development specialist, USA \\ ${ }^{3}$ Yasuj University of Medical Sci., Faculty of Medicine, Yasuj, Iran \\ ${ }^{4}$ Independent Researcher, Bangladesh \\ ${ }^{5}$ Hematology and Oncology, University of Aden, Yemen \\ ${ }^{6}$ Department of Medical \& Health Sciences for Woman, Peoples University of Medical and Health Sciences for Women, Pakistan \\ ${ }^{7}$ Biomedical laboratory, Citta Della Salute, Turin, Italy \\ ${ }^{8}$ President of IMA Academy
}

*Corresponding author: Mauro Luisetto, European Specialist Lab medicine, Branch General Toxicology- pharmacy and pharmacology IMA academy, Italy.

Received Date: October 08, 2019

Published Date: October 24, 2019

Abstract

Left and right colon cancer can be considered a really interesting model to deep understand what happen in a single apparatus and the related cancer phenomena. Environmental risk factors, hereditary syndromes and "sporadic" form added to 2 different kind of pathology (Left and right cancer) contribute to a real complex epidemiological situation. Aim of this work is to set 2 different kinds of "Toxic Pathologic" factors that make possible with their gradient the development of these 2 kinds of cancer in the same part of an apparatus.

Keywords: Colon cancer; LCRC; RCRC; Topography; Colon microbioma; Cancer genesis; Risk factors; Molecular biology

\section{Introduction}

Colorectal cancer (CRC) is a disease originating from the epithelial cells lining the colon or rectum of the gastrointestinal tract (GIT). Peters et al [1] written: "Colorectal cancer is a complex disease that develops as a consequence of both genetic and environmental risk factors. (3-5 \%) of cases arises from hereditary syndromes predisposing to early onset of CRC as a result of mutations in over a dozen well-defined genes. In contrast, CRC is predominantly a late onset "sporadic" disease, developing in individuals with no obvious hereditary syndrome". So, CRC follow a series of sequential genetic (acquired or inherited) and epigenetic alterations [1]. Colorectal cancer is a heterogeneous disease, exhibiting a variety of genetic and epigenetic alterations and present some relevant differences in Left vs right form. There are embryological origins, anatomical, histological, genetic, and immunological differences between right-sided colon cancer (RCC) and left-sided colon cancer (LCC).
This two-principal morphologic, multi-step pathways to CRC form: highly related to a MSI pathways ( hypermutated cancers ) or to CIN pathways (non-hypermutated) p-53 KRAS profile. But 3 different molecular pathways have been described for CRC.

1. MSI pathway: hyper-mutated cancers with high microsatellite instability, due to mutations in DNA mismatch repair system origin DNA proof-reading mechanism.

2. CIN pathway, high frequency of DNA copy number alterations, non-hypermutated tumors.

3. $\mathrm{CpG}$ island methylator phenotype (CIMP) is related to aberrant methylation in several genes, in sporadic cases with DNA mismatch repair pathway deficient (dMMR) and MSI-high tumors. BRAF mutations are also commonly observed within this last phenotype. 
Left colon cancer predominantly follow a CIN molecular pathway , instead right colon cancer form follows a MSI pathway. This kind of differences may reflect distinct biological characteristics of the proximal and distal colonic mucosa, acquired in embryonic or post-natal development, that determine a differential response to environmental factors. The differences in the epidemiology of proximal and distal CRCs could result from the presence of different kind pro-carcinogenic factors in the ascending vs descending colon, acting on cells with either similar or distinct biological characteristics.

According to J M. Carethers Gastroenterology 2011 [2]:"The colon is one organ, but develops from two different embryonic areas of the primitive gut: the midgut, which gives rise to the small intestine through to the proximal two thirds of transverse colon, and the hindgut, which gives rise to the distal third of the transverse colon through the upper anal canal. Functionally, the proximal colon absorbs most of the remaining water from the incoming digestive contents utilizing effective haustral shuttling, whereas the remainder of the colon utilizes peristalsis as its primary motility characteristic and lubricates the remaining waste as it becomes more solid while progressing toward the rectum [2]. The most widely recognized defines cancers proximal to the splenic flexure as right-sided and malignant growths at or distal to the splenic flexure as left-sided. This cut point is regularly utilized in light of the fact that most (proximal 66\%) of the transverse colon emerges embryologically from the midgut, and just the distal 33\% arises from the hindgut. Despite the fact that the midgut additionally offers ascend to different organs, including most of the small bowel and the vermiform appendix, the rare carcinomas arising from these organs have unique biologizes with different rates of key driver mutations and are clinically treated distinctly, and thus have not been included in most prior studies of CRC.

The right colon "sees" bile acids and other metabolites that have escaped the small intestine's reuptake or detoxifying mechanisms, and in a form before colonic bacterial action on the metabolites. Genetic profiling of normal right and left colon confirm major differences between sites of this endodermally derived organ: There are 165 genes showing 2-fold and 49 genes showing 3 -fold differences in gene expression between the adult human right and left colon. Females are more likely to develop proximal cancers whereas males develop more distal cancers, suggesting the possibility of hormonal influences on incidence. African Americans have higher prevalence of adenomas $9 \mathrm{~mm}$ proximally compared with Caucasians and have a higher prevalence of proximal cancers. Morphologic and genetic differences of colonic neoplasia are observed based on site within the colon. Laterally spreading tumors, a type of superficial "flat" spreading neoplasm, are commonly found in the right colon, and can be difficult to detect without enhanced endoscopic methods, these lesions are much more likely to contain cancer than their polypoid counterparts, and their lack of exophytic growth has been attributed to lack of KRAS mutation. Microsatellite instability (MSI), seen in up to $20 \%$ of sporadic cancers and prevalent in proximal cancers, is caused by hypermethylation of the DNA mismatch repair gene hMLH1 (through the CpG island methylator phenotype [CIMP]pathway), and these tumors lack mutated KRAS but contain the V600EBRAF mutation. Sessile serrated adenomas are associated with and progress to right-sided colon cancers, have BRAF mutation, and often manifest MSI from methylation of hMLH1, strongly suggesting that these mutations drive the development of these morphologically low profile and proximally located MSI tumors. All of these morphogenetic findings are likely unified in defining a pathogenic pathway in the right colon. One ascertained clinical consequence is that either the presence of CIMP or MSI in a patient's colon cancer predicts a lack of improved survival response with 5-fluorouracil- based chemotherapy. Detection of proximal serrated polyps appears to be endoscopist dependent, and their presence at screening increases interval neoplasia during surveillance ". The observing epidemiology of colon cancer and Irritable bowel syndrome (IBS) is possible to verify that there is difference between advanced countries and not advanced countries, from north part of the world vs south (diet/ Fiber). Is possible that a "gradient" in 2 different kind of "toxic factors" produce different kind of cancer in the same bowel tissue? Epidemiology , statistics, genetics and topography can be the right instrument to better understand of cancer phenomena. In this work of CRC, the epidemiology topography of lesions and the geographic distribution of cases make possible some global conclusion useful to better understand this neoplastic phenomenon [2].

According H Rafiemanesh: EXCLI J [3] "The incidence of colorectal cancer is different in the world about 20 times, and most of cases are available in Western industrial countries. The highest incidence of colorectal cancer was seen in Australia and New Zealand (39 per hundred thousand), West Europe (33.1 per hundred thousand), North America (30.1 per hundred thousand), and in East Asia, especially in Japan (18 per hundred thousand). Colorectal cancer was the lowest in Africa (3.6 per hundred thousand) and South Asia, including India and China" [3].

\section{Risk Factors}

According a meta analyses of colorectal cancer risk factors in 2013:

Johnson CM et al [4] written "Significant risk factors include inflammatory bowel disease ( $\mathrm{RR}=2.93,95 \%$ CI 1.79-4.81); CRC history in first-degree relative $(\mathrm{RR}=1.80,95 \% \mathrm{CI} 1.61-2.02)$; body mass index (BMI) to overall population ( $R R=1.10$ per $8 \mathrm{~kg} / \mathrm{m}(2)$ increase, $95 \%$ CI 1.08-1.12); physical activity ( $R R=0.88,95 \%$ CI $0.86-0.91$ for 2 standard deviations increased physical activity score); cigarette smoking ( $R R=1.06,95 \%$ CI 1.03-1.08 for 5 packyears); and consumption of red meat ( $R R=1.13,95 \%$ CI 1.091.16 for 5 servings/week), fruit ( $R R=0.85,95 \%$ CI 0.75-0.96 for 3 servings/day), and vegetables ( $\mathrm{RR}=0.86,95 \% \mathrm{CI}$ 0.78-0.94 for 5 servings/day). We developed a comprehensive risk modeling strategy that incorporates multiple effects to predict an individual's risk of developing CRC. Inflammatory bowel disease and history of CRC in first-degree relatives are associated with much higher risk of CRC. Increased BMI, red meat intake, cigarette smoking, 
low physical activity, low vegetable consumption, and low fruit consumption were associated with moderately increased risk of CRC." Cancer present some relevant peculiarity ; why some smokers not develop always pulmonary cancer and no smokers can show this pathology? (this in the same organ). Cancer is considered a pathology that come from genetics or environmental factors. But is interesting also other peculiarity: in observing epidemiology of colon cancer. The same tissue it must be present the same probability of cancer, but this often not happen, in and there is a Topographic VECTOR that must be deeply investigated. The same also the diet habits of humans in different geographic place gives interesting fact. As well as prevalence in advanced or not advanced region. Colorectal cancer originates from the epithelial tissue of the colon and present some relevant differences in LEFT vs RIGHT form. These 2 forms are related or to an MSI or to CIN p-53 KRAS genetic profile "Depending on the position, CRCs behave differently in terms of disease progression and overall survival. The difference between these tumors can be attributed to anatomical and developmental origin, or distinct carcinogenic factors (such as difference in bacterial population on the two sides of the colon, or exposure to distinct nutrients and bile acids, or a combination of both" [4]. Large bowel is the last part of the GI tract in vertebrates. (Water is absorbed here) and the remaining waste material is stored as feces before being removed by the act of defecation. The large intestine: the cecum, colon, rectum, and the anal canal. In humans, it is joined to the end of the small intestine at the cecum, via the ileocecal valve. (colon ascending, the transverse colon, and descending to the rectum, at the anal canal). It extracts water and salt from solid wastes before the elimination and is the site in which flora-aided (largely bacterial) fermentation of the un-absorbed material occurs. Unlike the small intestine, it does not play a major role in absorption of foods and nutrients. About 1,4- 1,6 liters of water arrives in the colon each day. The ascending colon including the cecum and appendix. The transverse colon including the colic flexures and transverse mesocolon. The descending colon including the sigmoid colon - The s-shaped region of the large intestine (Figure 1).

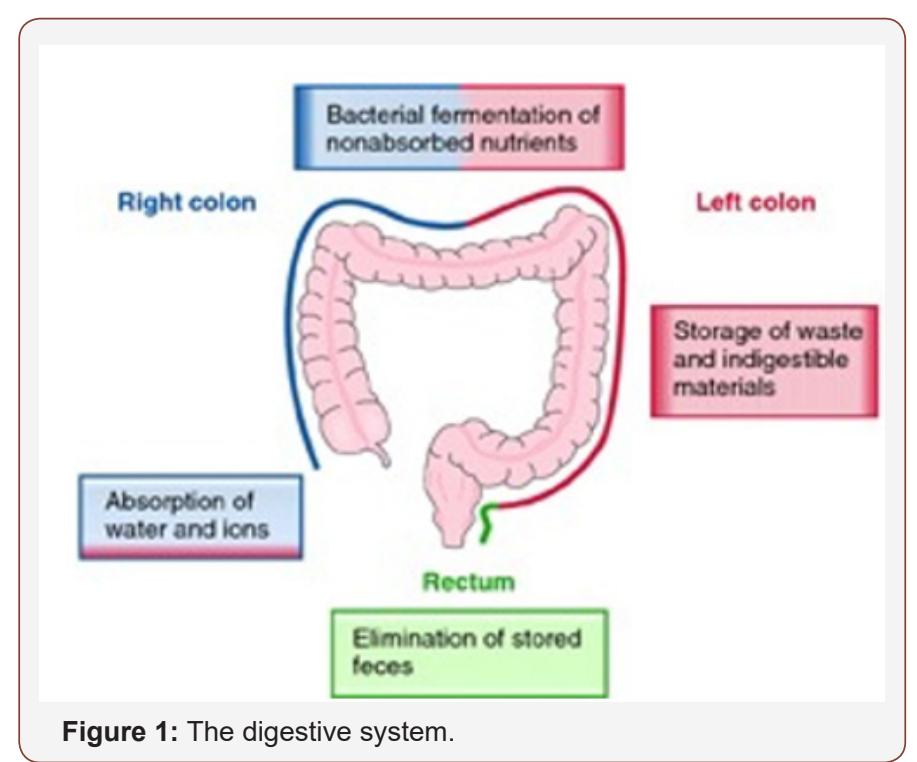

The parts of the colon are either intraperitoneal or behind it in the retroperitoneum. Retro-peritoneal organs more often do not have a complete covering of peritoneum, so they are fixed in location. Intra-peritoneal organs are completely surrounded by the peritoneum and are therefore mobile. the ascending colon, descending colon and the rectum are retro-peritoneal, the cecum, appendix, transverse colon and sigmoid colon are intraperitoneal. The cecum is the first section and is involved in the digestion, the appendix which develops embryo-logically from it, is a structure of the colon, and this is not involved in digestion process and considered to be part of the gut-associated lymphoid tissue. Appendix function: role in housing a sample of the colon's microflora, help to re-populate the colon with bacteria if the micro-flora has been damaged during immune reaction, a high concentration of lymphatic cells. The ascending colon: One of the main functions of the colon is to remove the water and other key nutrients from waste material and recycle it. It is some-times attached to the appendix via Gerlach's valve. In ruminants, the ascending colon is known as the spiral colon. Taking into account all ages and sexes, colon cancer occurs here most often (41\%). The descending colon functions: to store feces that will be emptied into the rectum. It is retroperitoneal in two-thirds of humans. In the other third, it has a (usually short) mesentery. The arterial supply comes via the leftcolic artery. Gut flora are very dense in this region. The walls of the sigmoid colon are muscular, and contract to increase the pressure inside the colon, causing the stool to move into rectum part. The sigmoid colon is supplied with blood from several branches' sigmoid arteries, branch of IMA. Blood supply: Arterial supply to the colon comes from branches of the superior mesenteric artery (SMA) and inferior mesenteric artery (IMA). Flow between these 2 systems communicates via a "marginal artery" that runs parallel to the colon for its entire length.

1. Venous drainage: The inferior mesenteric vein draining into the splenic vein, the superior mesenteric vein joining the splenic vein (to form the hepatic- portal vein.

2. Lymphatic drainage: from ascending colon and proximal $2 / 3$ of the transverse colon is to the colic lymph -nodes and the superior mesenteric lymph nodes, which drain into the cisterna chyli. The lymph from the distal one-third of the transverse colon, the descending colon, the sigmoid colon, and the upper rectum drain into the inferior mesenteric and colic lymph nodes.

Large intestine compacts feces and stores fecal matter in the rectum until it can be discharged via the anus in defecation. The large intestine also secretes $\mathrm{K}+$ and $\mathrm{Cl}$-. Recycling of various nutrients takes place in colon, and the fermentation of carbohydrates, short chain fatty acids, and urea cycling. The appendix contains a small amount of mucosa-associated lymphoid tissue which gives the appendix an undetermined role in immunity, important in fetal life because as it contains endocrine cells that release biogenic amines and peptide hormones.By the time the chyme has reached colon, most nutrients and $90 \%$ of the water have been absorbed by the 
body. Electrolytes like sodium, magnesium, and chloride are left as well as indigestible parts of ingested food (e.g., a large part of ingested amylose, starch which has been shielded from digestion heretofore, and dietary fiber). In the large intestine, most of water is removed, while the chyme is mixed with mucus and bacteria (known as gut flora) and becomes feces. The ascending colon receives fecal material as a liquid. The muscles of the colon then move forward and slowly absorb all the excess water, and gradually solidify as they move along into the descending colon. The bacteria use some fiber for their own metabolism and create acetate, propionate, and butyrate as waste products, which in turn are used by the cell lining of the colon for nourishment. In humans, about $10-15 \%$ of the undigested carbohydrate thus becomes available, though this may vary with diet; The $\mathrm{pH}$ in the colon varies between 5.6 and 7. Water absorption at the colon proceeds against a transmucosal osmotic pressure gradient. Intestinal Cells pump sodium ions into the intercellular space, raising the osmolarity of the intercellular fluid. The large intestine houses more then 800 species of bacteria with many of functions, like fungi, protozoa, and archaea. Species diversity varies by geography and by the diet behavior. The microbes in human distal gut often are more than 100 trillion (named today a kind of "forgotten organ"). The large intestine, in this place, absorbs products formed by the bacteria. Undigested polysaccharides (fiber) are metabolized to short-chain fatty acids by bacteria in the large intestine and absorbed by passive diffusion. The bicarbonate that the large intestine secretes acts to neutralize the acidity resulting from the fatty acids formations. Normal microbioma is also essential in the development of certain tissues, like the cecum and lymphatics. And involved in the production of cross-reactive antibodies. produced by the immune- system against the normal flora, also effective against related pathogens, preventing infection/invasion. The 2 most prevalent phyla of the colon are Bacteroides and firmicutes (Bacteroides are implicated in starting of colitis and colon cancer). A mucus layer protects the large intestine from attacks from colonic commensal bacteria.

\section{Epidemiology}

In 2012, CRC is the second most common cause of cancer in women (9.2\% of diagnoses) and the third most common in men (10.0\%) with it being the fourth most common cause of cancer death after lung, stomach, and liver cancer. It is more common in developed than developing countries. Incidences: vary 10-fold with highest rates in Australia, New Zealand, Europe and the US and lowest rates in Africa and South-Central Asia. Most colorectal cancers are due to old age and lifestyle factors, with only a small number of cases due to underlying genetic disorders. Risk factors include age, male sex, diet, obesity, high intake of fat, smoking, and lack of physical activity. (Dietary factors and other: red meat, processed meat, alcohol, sugar ,smoking, and a lack of physical exercise). Another risk factor is inflammatory bowel disease, which includes Crohn's disease and ulcerative colitis. Some of the inherited genetic disorders that can cause colorectal cancer include familial adenomatous polyposis and hereditary non-polyposis colon cancer; (less than 5\%). Starting as a benign tumor, (polyp), that over time becomes cancerous. CRC in developed countries is more common: more than $65 \%$ of cases. It is less common in women than men. More than $90 \%$ of CRC happen in patients with little or no genetic risk. Approximately $10 \%$ of cases are linked to insufficient activity. The risk from alcohol appears to increase at greater than 1 drink by day. Drinking 5- 6 glasses of water by day: is linked to a decrease in the risk of colorectal cancer and adenomatous polyps. Streptococcus gallolyticus is associated with colorectal cancer. Some strains of Streptococcus bovis/ Streptococcus equinus complex are consumed by millions of people daily and thus may be safe. $30-80 \%$ of people with Streptococcus bovis/gallolyticus bacteremia have concomitant colorectal tumors. People with IBD are at increased risk of colon cancer. The risk increases the longer a person has the disease, and the worse the severity of inflammation. People with IBD account for less than $2 \%$ of colon cancer cases yearly. In patient whit with Crohn's disease: $2 \%$ get colorectal cancer after 10 years, $8 \%$ after 20 years, and $18 \%$ after 30 years. Patient with a family history in 2 or more firstdegree relatives (such as a parent or sibling) have a 2 to threefold greater risk of disease and this group accounts for about $20 \%$ of all cases. Various of genetic syndromes are also associated with higher rates of CRC. The most common of these is hereditary nonpolyposis colorectal cancer (HNPCC or Lynch syndrome) which is present in about 3\% of people with colorectal cancer. Other include Gardner syndrome, and familial adenomatous polyposis (FAP). For patients with these syndromes, cancer almost always occurs and makes up $1 \%$ of the cancer cases. Mutations in the pair of genes (POLE and POLD1) have been associated with familial colon cancer form. Most of the deaths due to CRC are associated with metastasis. A gene that seem to contribute to the potential for metastatic disease, metastasis associated in colon cancer 1 (MACC1), has been isolated. (transcriptional factor that influences the expression of hepatocyte growth factor). This gene is associated with proliferation, invasion and scattering of colon cancer cells (in cell culture environment), and tumor growth and metastasis in mice model.

Epigenetic factors, like ab-normal DNA- methylation of tumor suppressor promoters, play a role in CRC. most frequently as a result of mutations in the Wet signaling pathway that increase signaling activity. The mutations can be inherited or acquired, and most probably occur in the intestinal crypt stem cell. The most commonly mutated- gene is the APC gene, that produces APC protein. (This prevents the accumulation of $\beta$-catenin protein). Without APC, $\beta$-catenin accumulates to high levels and trans-locates into nucleus, binds DNA, and this activates the transcription of protooncogenes. These genes play a crucial role for stem cell renewal and differentiation process, and if inappropriately expressed, they can produce cancer phenomena. Some cancers have increased $\beta$-catenin because of mutations in $\beta$-catenin (CTNNB1) that block its own breakdown or have mutations in other genes with function similar to APC such as AXIN1, AXIN2, TCF7L2, or NKD1. Other mutations must occur for the cell to become cancerous: in example the p53 protein, produced by the TP53 gene, normally monitors cell division and kills cells if they have Wnt pathway defects. A cell that acquire mutation in TP53 gene: transforms the tissue from a benign epithelial tumor into an invasive epithelial cell cancer. Other 
proteins involved in programmed cell death that are commonly deactivated in CRC are TGF- $\beta$ and DCC (Deleted in Colorectal Cancer). About $75 \%$ of all human genes are expressed in CRC , $1 \%$ of having increased expression in colorectal cancer compared to other forms of cancer. Some are oncogenes: over expressed in colorectal cancer. Genes encoding proteins like KRAS, RAF, and PI3K, that stimulate the cell to divide in response to growth factors, can acquire mutations that result in over-activation of cell proliferation. The chronological order of mutations is important. If a previous APC mutation occurred, a primary KRAS mutation often progresses to cancer rather than a self-limiting hyperplastic or borderline lesion. PTEN, a tumor suppressor, normally inhibits PI3K, but can sometimes become mutated and deactivated. CRC can be classified in: hypermutated and non-hypermutated tumor types. Non-hypermutated samples also contain mutated: CTNNB1, FAM123B, SOX9, ATM, and ARID1A. Progressing through genetic events, hyper-mutated tumors display mutated forms of ACVR2A, TGFBR2, MSH3, MSH6, SLC9A9, TCF7L2, and BRAF. The common fact among these kinds of genes, across both the tumor types, is their involvement in WNT and TGF- $\beta$ signaling pathways, which results in increased activity of MYC, a central player in colorectal cancer. Mismatch repair (MMR) deficient tumors are characterized by a relatively high amount of poly-nucleotide tandem repeats. This is due to a deficiency in MMR proteins - which are caused by epigenetic silencing and /or inherited mutations (Lynch syndrome). 15 \%of CRC show MMR deficiencies, with 3 percent developing due to Lynch syndrome. Mismatch repair system ROLE is to protect the integrity of the genetic code material into cells. A deficiency in MMR deficiency may lead also to an inability to detect / repair genetic damage, allowing for further cancer-causing mutations to occur and CRC to progress. The polyp to cancer progression sequence is a model of colorectal cancer pathogenesis. The polyp to cancer sequence describes the phases of transition from benign tumours into CRC over many years. (sequence are gene mutations, epigenetic alterations and local inflammatory changes).

Table 1: Difference Between Right Sided and Left Sided Colorectal Tumor [6].

\begin{tabular}{|c|c|}
\hline $\begin{array}{l}\text { Right Sided Colorectal } \\
\text { Cancer }\end{array}$ & Left Sided Colorectal Cancer \\
\hline $\begin{array}{l}\text { Mucinous adenocarcinomas, } \\
\text { sessile serrated adenomas }\end{array}$ & Tubular, villous adenocarcinomas \\
\hline Flat like morphology & Polypoid like morphology \\
\hline $\begin{array}{l}\text { MSI-high and mismatch repair } \\
\text { deficient tumors }\end{array}$ & CIN-high tumors \\
\hline $\begin{array}{l}\text { Highly immunogenic, high } \mathrm{T} \\
\text { cell infiltration }\end{array}$ & Low immunogenic \\
\hline $\begin{array}{l}\text { Metastases in peritoneal } \\
\text { region }\end{array}$ & Liver and lung metastases \\
\hline Occur in older ages & Occur in younger ages \\
\hline Predominantly occur in female & Predominantly occurs in males \\
\hline $\begin{array}{l}\text { Better prognosis at early } \\
\text { stages (stage I and II) }\end{array}$ & $\begin{array}{l}\text { Better prognosis at late stages (stage } \\
\text { III and IV) }\end{array}$ \\
\hline $\begin{array}{l}\text { Respond well to } \\
\text { immunotherapy }\end{array}$ & $\begin{array}{l}\text { Respond well to adjuvant } \\
\text { chemotherapies including standard } \\
\text { chemotherapies and targeted therapies }\end{array}$ \\
\hline
\end{tabular}

The term "field cancerization" was first used in 1953 to describe an area or "field" of epithelium that has been preconditioned (by what were largely unknown processes at the time) to predispose it towards development of cancer.(important in the progression process to CRC). More than $80 \%$ of the somatic mutations found in CRC tumors happen before the on-set of terminal clonal expansion, and more than half of this identified in tumors occurred in a preneoplastic phase (in a field defect), during the growth of normal cells. Epigenetic alterations present in tumors may have occurred in pre-neoplastic field defects. "etiologic field effect": not only molecular -pathologic changes in pre-neoplastic cells but also influences of exogenous environmental factors and molecular changes in the local micro-environment on neoplastic evolution from tumor initiation to death. Epigenetic alterations are much more frequent in CRC than the genetic (mutational) alterations. Vogelstein et al [5]: an average cancer of the colon has only 1 or 2 oncogene mutations and 1 to 5 tumor suppressor mutations ( "driver mutations"), with about 55 further "passenger" mutations. In addition to epigenetic alteration of expression of miRNAs, other epigenetic alterations in cancers that change gene expression levels include direct hypermethylation or hypomethylation of $\mathrm{CpG}$ islands of protein-encoding genes and alterations in histones and chromosomal architecture that influence gene expression.(150 hyper-methylations and 25 hypo-methylations of protein coding genes were frequently associated with CRC. Of the hypermethylated genes group , 10 were hyper-methylated in about $100 \%$ of colon cancers, many others in more than $50 \%$ of colon cancers. (11 hyper-methylations and 96 hypo-methylations of miRNAs were also associated with CRC). Abnormal (aberrant) methylation occurs as a normal consequence of the normal aging process and the risk of CRC cancer increases as increase the age (older). Half of the genes that show age-related methylation changes are the same genes that have been identified to be involved in the development of CRC. Epigenetic reductions of DNA repair- enzyme expression may likely lead to the genomic and epigenomic instability characteristic of cancer. For sporadic CRC, a deficiency in DNA repair activity is occasionally due to a mutation in a DNA repair gene but is much more frequently due to epigenetic alterations that reduce / silence expression of DNA repair genes. About half of colorectal cancer cases are due to some lifestyle factors, (so a quarter of all cases are preventable) [5]. Lifestyle risk factors with strong evidence include lack of exercise, cigarette smoking, alcohol, and obesity. Starting in the 1970s, dietary recommendations to prevent CRC included increasing the consumption of whole grains, fruits and vegetables, reducing the intake of red meat / processed meats. But In 2018 the National Cancer Institute written that "There is no reliable evidence that a diet started in adulthood that is low in fat and meat and high in fiber, fruits, and vegetables reduces the risk of CRC by a clinically important degree." With regard to dietary fiber, the 2014 WHO cancer report noted that it has been hypothesized that fiber might help prevent colorectal cancer, but most studies have not borne this out, and status of the science remained unclear. A 2019 review found evidence of benefit from dietary fiber and whole 
grains. The World Cancer Research Fund listed the benefit of fiber for prevention of colorectal cancer as "probable" (2017). Physical exercise is associated with a modest reduction in colon but not rectal cancer risk. High levels of physical activity reduce the risk of colon cancer by about $21 \%$.Sitting regularly for prolonged periods is associated with higher mortality from colon cancer. The risk is not negated by regular exercise, though it is lowered (Table 1)[6].

\section{Material and Methods}

With an observational approach some relevant (in our opinion) bio-medical literature (PUBMED) is analyzed to produce a new global conclusion. An experimental project hypothesis is then submitted to the researcher to better explain the concept.

\section{Result}

K So" Reide et al [7] said that "We observed significant inverse relationships between microsatellite instability and alterations in K-ras and p53.These inverse relationships were independent of tumor site and the type of microsatellite (mono- ,di-, or tetranucleotide repeat) used for instability analysis. Coding mononucleotide repeat mutations were significantly more common in unstable tumors than stable tumors. The molecular profile of colon cancers with microsatellite instability is therefore characterized by relatively infrequent mutations in K-ras and p53 and relatively frequent mutations in coding mononucleotide repeats. These different profiles of stable and unstable tumors most likely reflect different molecular pathways to sporadic colon cancer: the microsatellite stable (but chromosomally unstable) pathway, probably initiated by APC mutations, and the microsatellite instability pathway, in which early -catenin mutations are sometimes seen but in which the initiating event in most tumors is unknown These different molecular pathways and/or the specific genetic changes we report may in turn reflect different carcinogenic influences, such as diet or tobacco and alcohol use. Future studies that stratify colon cancers on the basis of these genetic changes may identify factors that contribute to one pathway or the other, relationships that might be obscured if the genetic heterogeneity of colon cancer is not taken into account" [7] (Figure 2).

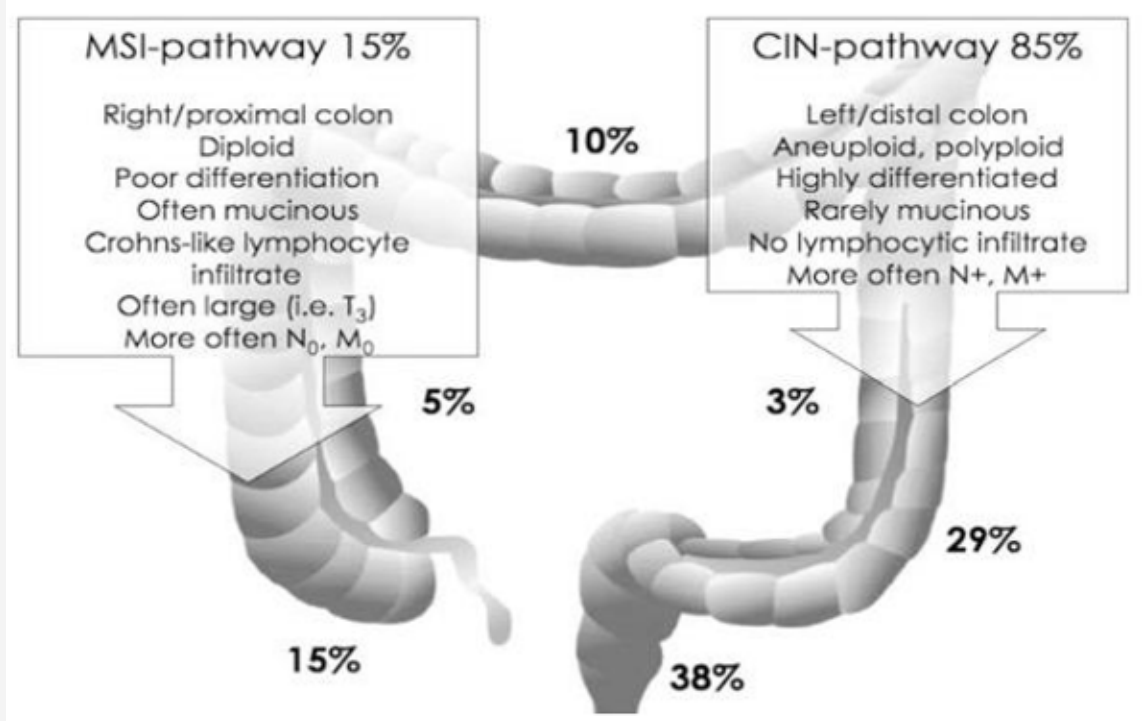

Figure 2: A simplified working-model of sporadic colorectal carcinogenesis: the CIN and MSI pathways [7].

Laura AE Hughes et al said that "It is now well accepted that CRC risk is highly modifiable through diet and lifestyle; recent reports suggest that up to $47 \%$ of CRC cases could be prevented by staying physically active, maintaining a healthy body weight and eating a healthy diet. The expert panel of the World Cancer Research Fund(WCRF), which is the organization responsible for publishing the most comprehensive review to date on risk factors related to diet and physical activity for cancer, has recently concluded that there is convincing strong evidence that body fatness, adult attained height, and consuming processed meat and alcoholic drinks increase the risk of developing CRC, while physical activity decreases the risk of developing CRC. CRC is not a single disease, but rather encompasses a heterogeneous complex of diseases characterized by numerous genetic and epigenetic abnormalities. There are two morphologic, multi-step pathways to CRC(the traditional adenoma-carcinoma pathway and the serrated neoplasia pathway), which are driven by three molecular carcinogenesis pathways (chromosomal instability
(CIN), microsatellite instability (MSI), and epigenetic instability (primarily the $\mathrm{CpG}$ island methylator phenotype (CIMP). Tumors arising via the traditional adenoma-carcinoma pathway begin as premalignant lesions comprising of conventional, tubular or tubulovillous adenomas, and account for approximately 60-90\% of sporadic CRCs. They are characterized by CIN, which describes a condition of aneuploidy that is caused by an accelerated rate of gains and losses of entire or large portions of the chromosome during cell division. CIN is associated with inactivating mutations or losses in the Adenomatous Polyposis Coli (APC) tumor suppressor gene, which occurs as an early event in this sequence. Mutations in the KRAS oncogene, as well as TP53,SMAD4, and PIK3CA genes are also frequently observed. With CIN, there is an increased rate of heterozygosity, which may contribute to the inactivation of tumor suppressor genes or activation of tumor oncogenes. Descriptively, tumors that arise from this pathway are more often associated with male sex and observed in the distal colon Approximately 10-30\% 
of sporadic CRC tumors arise via the serrated neoplasia pathway and have distinctly different histology compared to tumors derived from the traditional adenoma-carcinoma sequence. They are characterized by MSI, a form of genetic instability characterized by length alterations within simple repeated microsatellite sequences of DNA. This is the result of strand slippage during DNA replication, which is not repaired due to a defective post replication mismatch repair system. An early event of these tumors is mutation of the BRAF proto-oncogene, which inhibits normal apoptosis of colonic epithelial cells. The driving force of the serrated neoplasia pathway is the $\mathrm{CpG}$ methylator phenotype (CIMP), a form of epigenetic instability responsible for silencing a range of tumor suppressor genes, including MLH1. Loss of MLH1 is thought to cause microsatellite instability (MSI) and once MLH1 is inactivated, the rate of progression to malignant transformation is rapid. Descriptively, these tumors are more frequently associated with female sex, and are observed in the proximal colon" [8]. Inés Mármol et al [9] said that "Given its vast importance, it is not surprising that alterations in normal flora cause serious problems. For instance, the condition known as dysbiosis, in which the natural relationship between the host and the intestinal microbiota is disrupted, is considered to be one of the most probable causes of inflammatory bowel disease (IBD) or colorectal cancer (CRC). Many factors, such as antibiotic treatment or some types of diet, are known to be involved in the development of dysbiosis ".

Hiroko Nagao-Kitamoto et al [10] said that "A growing number of studies suggest that disturbance of the intestinal microbiota and its metabolic functions are strongly correlated with the initiation and progression of GI diseases, including functional dyspepsia, severe diarrhea, IBD, colorectal cancer (CRC), celiac disease, and IBS [10]. Ha Thi Nguyen et al [11] said that "Approximately $65 \%$ of CRC cases are sporadic with no family history or apparent genetic predisposition. The remaining cases are familial, arising from moderately penetrant inherited susceptibility, possibly interacting with environmental factors. The majority of sporadic CRCs ( $85 \%)$ exhibit chromosomal instability (CIN), with changes in chromosome number and structure. These changes include gains or losses of chromosomal segments, chromosomal rearrangements, and loss of heterozygosity ( $\mathrm{LOH}$ ), which results in gene copy number variations (CNVs). These alterations affect the expression of tumor associated genes, and/or genes that regulate cell proliferation or cell cycle checkpoints, which, in turn, may activate pathways essential for CRC initiation and progression. The remaining sporadic cases $(\sim 15 \%)$ have high frequency microsatellite instability (MSI) phenotypes. hereditary CRC has two well described forms: Familial adenomatous polyposis (FAP) $(<1 \%)$ patients inherit a mutated copy of the adenomatous polyposis (APC) gene, whereas hereditary non-polyposis colorectal cancer (HNPPC, or Lynch syndrome) (1-3\%) is characterized by MSI, a consequence of a defective DNA mismatch repair (MMR) system. The other forms of hereditary CRC include a rare syndrome called hamartomatous polyposis syndrome $(<1 \%)$ and the common inherited cases caused by less penetrant inherited mutations (32\%) [9]. Sequential acquisition of genetic and epigenetic alterations is well defined and confirmed to drive the initiation and progression of adenomas to carcinomas in sporadic and inherited forms of CRC. Generally, CRC formation begins with the transformation of a normal colorectal epithelium to a benign adenoma, and then progresses through the stepwise accumulation of multiple genetic and epigenetic aberrations, subsequently leading to invasive and metastatic tumors. This process may take years to decades to escape the multiple regulatory layers of the cells and to fully There are three major pathways associated with CRC pathogenesis, namely: CIN, MSI and CpG island methylator phenotype (CIMP)" (Figure 3).

Chromosomal instability (CIN)

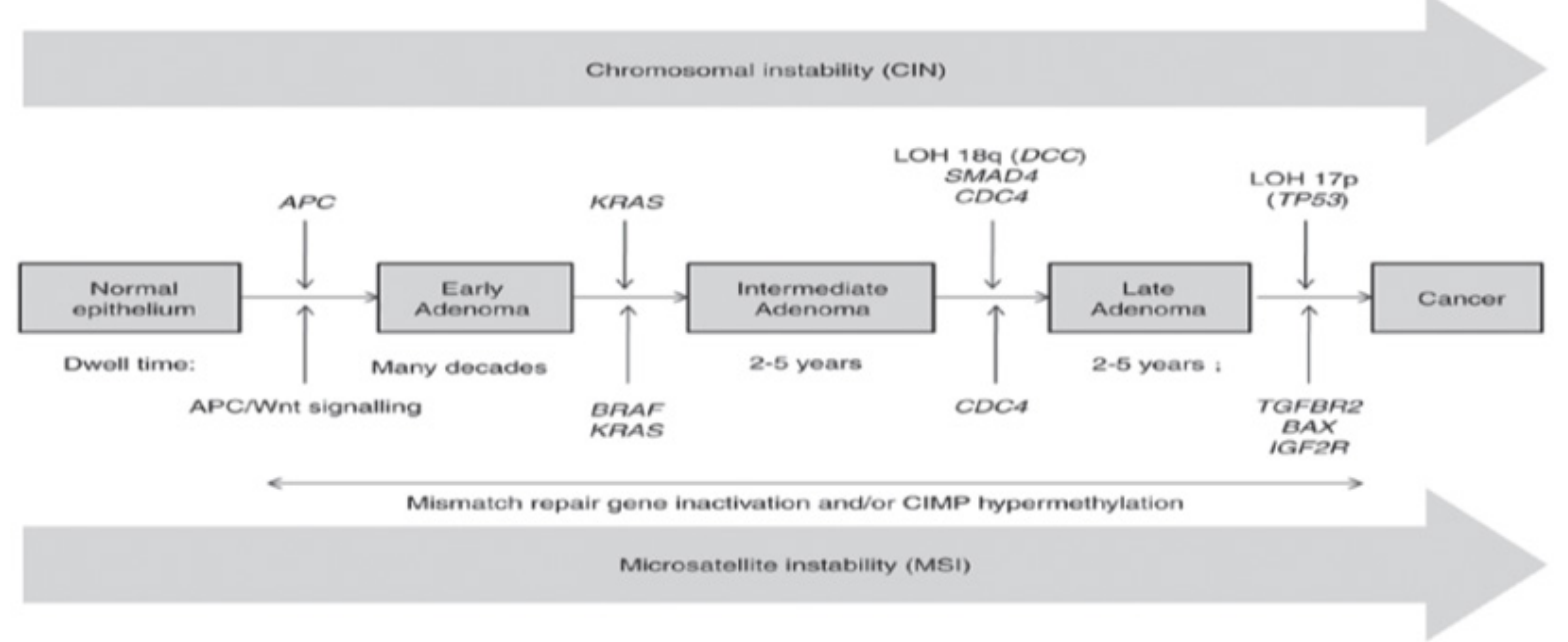

Figure 3: Colorectal adenoma-carcinoma sequence. The APC mutation is the first step transforming normal colorectal epithelium to adenoma. The adenoma-carcinoma sequence is caused by three major pathways: CIN, MSI and CIMP. CIN, Chromosomal instability; MSI, microsatellite instability; CIMP, CpG island methylator phenotype; APC, adenomatous polyposis; KRAS, KRAS proto-oncogene GTPase; BRAF, B-Raf proto-oncogene serine/threonine kinase; TP53, tumor protein 53; LOH, loss of heterozygosity; HNPPC, hereditary non-polyposis colorectal cancer; MLH1, mutL homolog 1; MSH2, mutS homolog 2; DCC, DCC netrin 1 receptor; TGFBR, transforming growth factor- $\beta$ receptor; BAX, BCL2 associated $X$ apoptosis regulator; IGF2R, insulin like growth factor 2 receptor; CDC4, cell division control protein 4 Modified of HA THI NGUYEN1 and HONG-QUAN DUONG [11].

Citation: Mauro Luisetto, Behzad Nili Ahmadabadi, Hossein Nili-Ahmadabadi, Ahmed Yesvi Rafa, Gamal Abdul Hamid, et al. Comparison of Risk Factors and Molecular Analysis of Right-Sided Colon and Left Sided Colon Cancer. Adv Can Res \& Clinical Imag. 2(2): 2019. ACRCI. 


\section{Epidemiological perspective}

According to Burcin Barana et al [6] People aged 50 or above have an increased risk of developing CRC. More than $90 \%$ of people diagnosed with CRC are over 50 years of age; CRC incidence among the younger people is likely to increase in recent years. Interestingly, comparison between stage III and IV RCRC and LCRC patients exhibited a poor overall survival of RCRC patients, while stage I and II RCRC patients exhibited a better overall survival than LCRC patients. According to different cohort studies, RCRC occurs predominantly in females and older people, and LCRC occurs predominantly in males, and at an early age., it should be noted that LCRC also occurs after 50 years of age, with a frequency more than that of RCRC. Hereditary cancer syndromes except familial adenomatous polyposis (FAP) tend to occur in the right side of the colon. Hereditary nonpolyposis colorectal cancer (HNPCC) or Lynch syndrome is the most common form of hereditary CRC, observed in $1-6 \%$ of all CRC cases. Most of the CRC (about 70\%) tend to occur in the left side, whereas, a small percentage (about 10\%) occurs in the right side. While RCRC tumors tend to be MSI-high, LCRC tumors are CIN-high tumors.

\section{Anatomical and histological perspective}

The colon is approximately $150 \mathrm{~cm}$ in length and extends from the ileocecal valve up to the anus. It is composed of seven parts including cecum, ascending colon, transverse colon, descending colon, sigmoid colon, rectum, and anus. This distinction between right-sided and left-sided colon is based on their embryological origins. The cecum, appendix, ascending colon, hepatic flexure, and proximal two thirds of the transverse colon have originated from the midgut, whereas distal one third of the transverse colon, splenic flexure, sigmoid colon, descending colon and rectum have originated from the hindgut. Hence, the right-sided CRC (RCRC) tumors arise from ascending colon, and proximal two thirds of the transverse colon and the left-sided CRC (LCRC) tumors arise from the descending and sigmoid colon, and distal one third of the transverse colon. Besides the difference in their origin, these tumors exhibit different histology. While right-sided tumors show sessile serrated adenomas or mucinous adenocarcinomas, leftsided tumors show tubular, villous and typical adenocarcinomas. Right-sided CRC patients tend to have advanced and bigger tumors, which are often poorly differentiated. The genomic make-up of RCRC and LCRC are totally different from each other. While RCRC patients tend to have more microsatellite instability-high (MSIhigh) tumors, LCRC patients tend to have chromosomal instabilityhigh (CIN-high) tumors. Right-sided CRC patients tend to have advanced and bigger tumors, which are often poorly differentiated. The genomic make-up of RCRC and LCRC are totally different from each other. While RCRC patients tend to have more microsatellite instability-high (MSI-high) tumors, LCRC patients tend to have chromosomal instability-high (CIN-high) tumors. Microsatellite instability-high tumors are characterized by the mutations or inactivation of the DNA mismatch repair system, and consequently single nucleotide mutations and alterations in the length of repetitive microsatellite sequences in the genome are observed in these tumors. Sessile serrated adenomas (SSA) are premalignant lesions with abnormal proliferation, crypt distortion, and dilation. Sessile serrated adenomas can be distinguished from the traditional serrated adenomas due to the lack of cytological dysplasia in their early stage. They are frequently observed in RCRC and MSIhigh tumors. Mucinous adenocarcinomas, another type of CRC, are commonly observed in RCRC and MSI-high tumors. Mucinous adenocarcinomas are characterized by excessive mucin excretion. Mucinous cancers have faster progression when compared with adenomatous polyps and are commonly diagnosed in inflammatory bowel disease patients MSI-high tumors have more T cell infiltrates because these tumors carry more immunogenic mutations and harbor increased number of neoantigens. Histologically, many MSI-high tumors have shown Crohn's-like lymphoid reaction in the invasive front of the tumor. Presence of T cells in these tumors is associated with better prognosis and less frequent metastasis.

Approximately $70-85 \%$ of CRCs develop via the traditional pathway, which is also known as CIN pathway. Chromosomal instability is characterized by widespread imbalances in chromosome number (aneuploidy) and loss of heterozygosity (LOH). In this classical model, the early lesions detected are tubular or villous or tubulovillous adenomas. Most of the adenomas in the CIN pathway are adenomatous polyps, but only $<5 \%$ harbor malignancy potential. villous adenomas, comprising only $5-10 \%$ of polyps have a high potential (35-40\%) of malignancy. The presence of villous polyps in the patient is the determining factor for the risk of malignancy in patients. These polyps turn into carcinomas in 10 to 20 years owing to the accumulation of sequential mutations. Hence, it is essential to excise these polyps by polypectomy in the early stages to reduce the risk of CRC. Metastasis of CRC significantly affects the overall survival among the patients. Distant metastases are present in approximately $25 \%$ of patients; diagnosis and respectability of metastasis significantly dictates the outcome. The sites of metastasis differ between LCRC and RCRC patients. While LCRC patients tend to have liver and lung metastasis, RCRC patients have peritoneal carcinomatosis. Historically peritoneal metastases had the worst outcome; with the advance in hyperthermic intraperitoneal chemotherapy (HIPEC), the survival rates have improved among this patient group. Colorectal cancer is the third most common cancer worldwide with a high mortality rate at the advanced stages. colorectal cancer is not a single type of tumor; its pathogenesis depends on the anatomical location of the tumor and differs between right side and left side of the colon. Tumors in the proximal colon (right side) and distal colon (left side) exhibit different molecular characteristics and histology. In the right-sided tumors, mutations in the DNA mismatch repair pathway are commonly observed; and these tumors generally have a flat histology. In the left-sided tumors, chromosomal instability pathway-related mutations, such as KRAS, APC, PIK3CA, p53 mutations are observed and these tumors demonstrate polypoidlike morphology. Therapy responses are totally different between these tumor entities. Left-sided colorectal cancer (LCRC) patients 
benefit more from adjuvant chemotherapies such as 5-fluorouracil (5-FU)-based regimes, and targeted therapies such as antiepidermal growth factor receptor (EGFR) therapy and have a better prognosis. Right-sided colorectal cancer (RCRC) patients do not respond well to conventional chemotherapies but demonstrate more promising results with immunotherapies because these tumors have high antigenic load. For the development of effective therapy regimes and better treatment options, it is essential to evaluate right-sided and left-sided tumors as separate entities and design the therapy regime considering the differences between these tumors. Left-sided CRC predominantly follow the CIN molecular pathway. Fearon and Vogelstein first proposed the multistep genetic model for colorectal carcinogenesis, which is known as traditional pathway (CIN pathway). In this model, carcinogenesis begins with the inactivation or deletion of adenomatous polyposis coli (APC) tumor suppressor gene, followed by the activation of Kirsten ras homolog (KRAS) oncogene, the inactivation of p53 tumor suppressor gene and other mutations. Right-sided CRC predominantly characterized by microsatellite instability and are hypermutated tumors. Deficient mismatch repair/microsatellite instability-high (dMMR/MSI-high) are caused by defects in the DNA mismatch repair system, which further accelerates the accumulation of single nucleotide mutations, and alterations in the length of repetitive microsatellite sequences in the genome. Most HNPCC tumors, and a portion of non-hereditary CRCs showed high dMMR/MSI status.

Colorectal cancer is the third most common cancer with high mortality rates in the advanced stages. Although the detection of CRC in the early stages is possible with routine colonoscopy screening, the prevalence is still increasing, especially in developing countries. Notably, CRC is not one type of disease, rather it acts as two different diseases in the same organ. The behavior of CRC is highly affected by the anatomical location of the tumor, which in turn affects its molecular and immunological characteristics. Understanding the characteristics of these two different entities is very important for developing effective therapies. Right-sided CRC tumors are commonly microsatellite instable tumors, whereas, LCRC tumors are chromosomal instable tumors. The conclusion found that rightand left-sided colon cancers are significantly different regarding epidemiological, clinical, and histological parameters. Patients with right-sided colon cancers have a worse prognosis. These discrepancies may be caused by genetic differences that account for distinct carcinogenesis and biological behavior ". Camille Thélin et al [12] said that Symptoms depend somewhat on the site of the tumor. In general, right-sided colon cancers are usually detected at an advanced stage with severe symptoms. In general, the right-sided colon cancers are commonly larger, producing vague abdominal discomfort and sometimes a palpable mass. Obstruction is rarely a presenting symptom, as the diameter of the right colon is larger than the left colon. If the tumor involves the cecum, it could block the ileocecal valve causing small bowel obstruction. Those with right-sided colon cancers are significantly older and are predominantly women (46\% women versus 38\% men). Because of higher rates of comorbidities, survival is worse in those with right-sided carcinomas. Left-sided colon cancers and rectal cancers tend to arise in younger, male populations with high-incidence risk. Cancers involving this portion of the bowel produce symptoms that range from obstruction to tenesmus, to alternating constipation and diarrhea with pencil-thin stools. Often, there is blood witnessed either in the stool or coating the stool, in comparison to the right-sided colon cancers. Similarly, rectal cancers can cause obstruction and similar types of bowel movement changes as the left-sided carcinomas. Modifiable risk factors include diet, physical activity, weight, cigarette-smoking, and alcohol intake. Other modifiable risk factors include low calcium content, low selenium content, and very low salt intake. Occupational hazards, such as asbestos exposure, have been linked to increased risk of colorectal cancer when compared to the rest of the general population. Socioeconomic factors, along with access to (and use of) health care services, are also important contributing risk factors. there is a disproportionately high incidence of colorectal cancers in low socioeconomic status populations. Non modifiable risk factors associated with higher risk of colorectal cancer include increasing age, personal history of adenomatous polyps, personal history of inflammatory bowel disease, genetic inheritance, race/ethnicity, and gender. Unlike modifiable risk factors that could theoretically have been avoided, these risk factors are not considered part of the "environmental nature" of this disease. Thus, they are not controllable. They do, play an important role in screening and identifying susceptible patients.

\section{Modifiable risk factors: Diet}

Diets associated with high incidence of colorectal cancer include diets with high consumption of red or processed meat, diets high in fat, beer-drinking, diets low in calcium intake, and diets low in whole-grain fiber, fruits and vegetables. This represents a typical "Western diet." On average, $40-45 \%$ of Western diets have total caloric intake made up from fatty foods (including meat products), while fat only accounts for about $10-15 \%$ of dietary makeup in lower-risk populations-China, India, and parts of Africa and South America. Consequently, it has been shown that the developed world carries the majority of the burden (Australia, New Zealand, Canada, the United States and parts of Western Europe), likely due to similarity in lifestyles and diets. The hypothesis behind dietary fat as a risk factor is that the fat enhances hepatic cholesterol and bile acid synthesis resulting in increased sterols in the colon. Those sterols are then converted into secondary bile acids, cholesterol metabolites, and potentially toxic metabolic compounds. While the exact pathogenesis remains unknown, what is known is that these sterols and bile acid metabolites cause damage to colonic mucosa, thus enhancing proliferative activity which could lead to dysplasia. This has been demonstrated in animal models, where animals fed polyunsaturated and saturated fats have higher numbers of adenocarcinoma than those on a low-fat diet. This has also been shown in human population studies where those with colorectal cancer tend to have higher fecal bile acid levels, while a recent metaanalysis has shown that consumption of red meat and processed 
meat is positively associated with risk of both colon- particularly the descending and sigmoid colon- and rectal cancer. The "Western diet" also comprises of lower amounts of fiber intake. Multiple epidemiology studies have shown a geographical difference of lower colorectal cancer incidence rates in places with higher fiber intake. It is even postulated that due to the ability of fiber to change the colonic pH, carcinogenesis may be impeded. Dietary fiber also increases fecal bulk, thus diluting the aforementioned carcinogenic compounds and reducing transit time and mucosal contact. In fact, fiber has been found to decrease the concentration of sterol and bile acid metabolites that could be implicated in creating carcinogenic compounds. Again, this has been demonstrated in animal models, where Epidemiology of Colorectal Cancer- Incidence, Lifetime Risk Factors Statistics and Temporal Trends increased fiber intake led to decreased concentration of specific bacterial metabolic enzymes that could be implicated in creating carcinogenic compounds. Unfortunately, for all its experimentally demonstrative protective roles, increased fiber supplementation has been unable to prevent adenoma recurrence in several randomized-controlled trials.

\section{Modifiable risk factors: physical activity and body weight}

Other modifiable risk factors are physical inactivity and excess body weight. Decreased gut motility, increased insulin resistance, lower metabolic rates, and increased circulating estrogens are all mechanisms implicated in the higher risk of colorectal cancer associated with this modifiable risk factor.

\section{Modifiable risk factors: social behaviors}

Associated with a higher risk is regular consumption of cigarettes and alcohol. Carcinogenic metabolites found in both tobacco and alcohol are considered promoters of tumor growth, based on experimental studies in animals. Cigarette-smoking has been attributed to $12 \%$ of colorectal cancer deaths, while alcohol consumption has been linked with early onset colorectal cancers, specifically tumors in the distal colon. There is information showing that there is higher risk in active smokers for development of rectal cancer.

\section{Nonmodifiable risk factors: age}

Increasing age carries a higher likelihood of colorectal cancer, specifically after the age of 40 . Cancer incidence rises progressively after the age of 40 in the general population, with $90 \%$ of colorectal cancers occurring in those aged 50 years and older. In fact, a 50year old has $5 \%$ chance of developing cancer and $2.5 \%$ chance of dying from this cancer after the age of 80 years. As such, the US Preventative Task Force (USPSTF) has defined "average risk" as those aged 50 years or more with no personal history of colorectal cancer or adenomas, no inflammatory bowel disease, and with negative family history. Put in other terms, the incidence rate is more than 50 times higher in those 60-79 years old than in those less than 40 years old. In contrast, those with "increased risk" include those with a personal history of colorectal cancer, personal history of colonic adenomas, family history of sporadic colorectal cancer, as well as family history of sporadic adenoma. Finally, those with "high risk" include those with hereditary nonpolyposis colorectal cancer (Lynch syndrome), polyposis syndromes, and inflammatory bowel diseases (IBD) [10].

\section{Nonmodifiable risk factors: history of colonic adenomatous polyps}

Carrying a personal history of adenomatous polyps has an increased risk of developing colorectal cancer, in comparison to those with no history of adenomas. In recent literature, it Is screening for Colorectal Cancer with Colonoscopy was reported that $95 \%$ of sporadic colorectal cancers developed from such adenomas, usually after a protracted period, which has been estimated anywhere from 5 to 10 years. while nearly all colorectal cancer arises from adenomas, only a small minority of these dysplastic polyps actually progress to cancer (5\% or less).

\section{Nonmodifiable risk factors: family history of colonic adenomatous polyps or colorectal cancer}

The majority of cases occur in those with family history of either colorectal cancer or adenomatous cancer. In fact, there is a two- to three-fold increased risk of sporadic cancer in those with firstdegree relatives. This means that up to $20 \%$ of those with colorectal cancer have family members affected by this disease. This risk becomes even higher when there are two or more relatives involved and when those family members are affected by the disease at an age younger than 60 .

\section{Nonmodifiable risk factors: hereditary polyposis conditions}

Those with recognized inherited polyposis syndromes carry an even higher risk. Recent literature estimates that about 5-10\% of sporadic colorectal cancers are the outcome of inherited conditions, such as the familial adenomatous polyposis (FAP) and hereditary nonpolyposis colorectal cancer (HPNCC). HPNCC (also called Lynch syndrome) is thought to comprise of about $1-6 \%$ of all colorectal cancers. It carries a lifetime risk of cancer as high as $70-80 \%$. FAP and its variants account for less than $1 \%$ of all colorectal cancer cases, but almost all those diagnosed with this disorder will develop cancer if the colon is not removed by the age of 40. Other hereditary conditions that are associated with sporadic colorectal cancers include Gardner's syndrome (highrisk), Turcot's syndrome (high-risk), and Peutz-Jeghers syndrome (low-to-moderate risk). Appropriate screening recommendations are made for this population subtype, which will not be discussed here.

\section{Nonmodifiable risk factors: personal history of Inflammatory Bowel Disease (IBD)}

Those with IBD- ulcerative colitis and Crohn's disease ,also carry an increased risk of developing colorectal cancer. It has been estimated that the relative risk of colorectal cancer in patients with IBD ranges from 4- to 20 -fold. Thus, appropriate screening recommendations are made for this population subtype, which will not be discussed here". 


\section{Environmental Differences}

Lee GH et al [13] said that Somatic genetic and epigenetic changes seen in CRC can be attributed to the environmental effect on the colonic tissue. Anatomically, the content and variety of microbiota differs from proximal and distal colorectum. Fermentation reactions produce short-chain fatty acids at significantly higher levels in the distal colorectum compared to the proximal colon. The higher bacterial load in the distal colorectum results in more hydrolytic and reductive bacterial enzymes involved in production of mutagenic metabolites than found more proximally. Pro-mutagenic and pro carcinogenic metabolites such as N-nitroso compounds(NOC), which could potentially lead to increased DNA. 1000 genes showing differences in expression relevant to major signaling pathways involved in CRC pathogenesis. About $70 \%$ of these genes were expressed at higher level in the distal colon and $30 \%$ in the proximal colon. Further analysis confirmed that there is higher transcriptional activity in the distal colon, which may explain some of the characteristics of LCRC. According to a Scandinavian twin study, around $35 \%$ of CRC cases can be attributed to heritable factors, but only $2 \mathrm{e} 6 \%$ are due to high penetrance mutations in genes such as APC, MLH-1 and MSH-2.20e23 Such penetrance mutations in mismatch repair genes or other oncogenes are relatively rare, and it is likely that most familial CRC is due to low penetrance polymorphism of other genes. Low penetrance polymorphisms could either lead directly to carcinogenesis or facilitate the progression of carcinoma. Prevalence of CRC in adenomatous polyposis syndromes differs depending on the colonic subsites. Patients with familial adenomatous polyposis (FAP) have been shown to have higher prevalence of distal colonic cancers, whereas CRC in MUTYH associated polyposis is more frequent in proximal colon. Historically, Delattre described that almost all distal colonic cancers showed allelic losses at chromosomal regions in $17 p$, 18 and $5 q$ e in line with genetic alterations in tumor suppressor genes.26 More recently, Breivik demonstrated that KRAS and p53 mutations were characteristic of LCRC but seen more rarely in RCC, which were predominantly microsatellite instability (MSI) positive tumors. Soong et al. summarized findings from 13 previous studies comparing p53 mutations in RCC and LCRC, demonstrating higher proportion of p53 positive tumors in distal colorectum compared to proximal colon. Environmental differences somatic genetic and epigenetic changes seen in CRC can be attributed to the environmental effect on the colonic tissue. Anatomically, the content and variety of microbiota differs from proximal and distal colorectum. Fermentation reactions produce short-chain fatty acids at significantly higher levels in the distal colorectum compared to the proximal colon. The higher bacterial load in the distal colorectum results in more hydrolytic and reductive bacterial enzymes involved in production of mutagenic metabolites than found more proximally. Pro-mutagenic and procarcinogen metabolites such as N-nitroso compounds (NOC), which could potentially lead to increased DNA alkylation, have also been found at higher levels in the distal colorectum compared to the proximal colon in a cohort of CRC patients. There are also differences in intensity of exposure to bile acids leading to differences in metabolism of bile acids along the colon. Studies have shown that a Western lifestyle, particularly diet, leads to increased secretion of bile acids. High-fat diet changes the pattern of hepatic bile acid secretion resulting in an increase in concentration of deoxycholic and lithocholic acid in bile. Both animal and human studies have shown a potential role of bile acids in promoting tumorigenesis. A recent study from Lax showed that the expression of the foresaid-X receptor, which plays a key role in intestinal defense against toxic bile acids, gradually decreases from the proximal to distal colon (excluding rectum) in the healthy gut. However, in CRC, RCC showed an inverse correlation between FXR expression and histopathological tumor grade, not seen in LCRC, suggesting differences in the role of bile acids in pathogenesis between RCC and LCRC. Series of meta-analyses by Larsson et al. give a good summary of the relationship between other environmental risk factors such as obesity, diabetes and meat consumption and CRC. Populations with the highest meat consumption have an increased risk of CRC, and this risk id greater in development of rectal cancer compared to colon cancer $[14,15]$.

Three studies specifically correlated meat consumption and CRC development according to subsite and concluded that high consumption of red meat is associated with an increased risk of LCRC, but not of RCC.

Larsson et al. analyzed 30 prospective studies in their metanalysis on the relationship between obesity and risk of CRC development This study concluded that obesity is associated with increased risk of CRC in both men and women. obesity is only associated with increased risk of rectal cancer in men, but not in women. Overall analysis concluded that increased body mass index was more strongly correlated to increased risk of colon cancer than to rectal cancer. Type 2 diabetes is another independent risk factor associated with CRC, demonstrated by numerous epidemiological studies. Recent studies have shown that diabetic patients have a poorer outcomeafter CRC treatment. Larsson etal [16] meta-analysis demonstrated that despite an overall increase in relative risk of developing CRC in diabetic patients of 30\%, there was no difference between RCC and LCRC. Genetic and epigenetic differences CRC is a disease caused by genetic alterations, which involves alterations in the DNA of oncogenes and/or tumor suppressor genes. changes in gene expression can occur via the regulatory portions of DNA or messenger RNAs without changes in the actual DNA sequence, referred to as "epigenetic" changes.18 Alteration in DNA methylation is thought to be a major contributing cause of epigenetic changes. Already, there are underlying gene expression differences between the proximal and distal colon before development of cancer. This appears to be present prior to birth according to Glebov et al. [17], but in adulthood, there are over1000 genes showing differences in expression relevant to major signaling pathways involved in CRC pathogenesis. About 70\% of these genes were expressed at higher level in the distal colon and $30 \%$ in the proximal colon. Further analysis confirmed that there is higher transcriptional activity in the distal colon, which may explain some of the characteristics 
of LCRC. According to a Scandinavian twin study, around 35\% of CRC cases can be attributed to heritable factors, but only2e6\% are due to high penetrance mutations in genes such as APC, MLH-1 and MSH-2.20e23 Such penetrance mutations in mismatch repair genes or other oncogenes are relatively rare, and it is likely that most familial CRC is due to low penetrance polymorphism of other genes. Low penetrance polymorphisms could either lead directly to carcinogenesis or facilitate the progression of carcinoma. Prevalence of CRC in adenomatous polyposis syndromes differs depending on the colonic subsites. Patients with familial adenomatous polyposis (FAP) have been shown to have higher prevalence of distal colonic cancers, whereas CRC in MUTYH associated polyposis is more frequent in proximal colon. Historically, Delattre et al. described that almost all distal colonic cancers showed allelic losses at chromosomal regions in $17 \mathrm{p}, 18$ and $5 \mathrm{q}$ e in line with genetic alterations in tumor suppressor genes.

More recently, Breivik et al. demonstrated that KRAS and p53 mutations were characteristic of LCRC but seen more rarely in RCC, which were predominantly microsatellite instability (MSI) positive tumors. Soong et al. summarized findings from 13 previous studies comparing p53 mutations in RCC and LCRC, demonstrating higher proportion of p53 positive tumors in distal colorectum compared to proximal colon. Immunological differences. There are distinct differences in mucosal immunology between the compartments of the colorectum. A study by Paski demonstrated that in the healthy adult colorectum, there is significantly increased immune activity in the cecum compared to the rectum. Histological evidence of inflammation was greatest in the cecum, with higher epithelial injury, lamina propria cellularity, cryptitis and over all activity and chronicity score for inflammation in cecum compared to rectum. Additionally, the mucosal eosinophil count was also highest in the cecal mucosa, which is also demonstrated in pediatric colorectum. Intraepithelial T-cells were also shown to be higher in the proximal colon compared to distal colorectum in healthy adults. Taken together, there is a negative gradient of various immune cells from the proximal colon to distal colorectum, which may be due to both inherited and environmental factors. These differences in mucosal immunology between proximal and distal colorectum could be explained by the differences in gut microbiota. Studies have shown that the human colon contains complex and diverse microbial colonies of approximately 1013 to 1014 bacteria, and these numbers increase with a positive gradient from the proximal colon to distal colorectum. Mucosal immune cells in the colorectum have to maintain a delicate balance between immunogenicity against pathogens and tolerance for the commensal microbiota. Hypothetically, the distal colorectum, with the highest concentration and numbers of microbiota, would require immune cells to promote tolerance rather than immunogenicity. Therefore, one might speculate that mucosal immune cells in the proximal colon would demonstrate increased immunogenicity compared to those in the distal colorectum. This could potentially explain the differences in types and numbers in immune cells between proximal and distal colorectum. thus far, there has been no study in which differences in immune activity between the proximal and distal colorectum have been directly compared. Applying these differences in mucosal immunology in the context of CRC, tumors developing in the proximal colon would be exposed to increased immune activity compared to LCRC. It is possible that the underlying immunological differences could explain the characteristic differences in pathogenesis and outcome between RCC and LCRC.

MSI positive tumors arise more commonly in the proximal colon, and the presence of tumor infiltrating lymphocytes(TIL) is one of its characteristics. Additionally, Ghazi et al's analysis over 1600 colorectal tumors showed TIL was significantly more common in proximal colon cancer on multivariate analysis after considering other morphologicalfeatures.17 Studies have also shown that the presence and density of TIL within colorectal tumors is associated with better overall prognosis, which could be partially explained by the more "active" mucosal immunology in the proximal colon compared to tolerogenic distal colorectum. Galon suggested that determination of the type, density and location of immune cells in CRC predicted overall survival better than, and independent of, the widely used UICC-TNM staging, emphasizing the importance of mucosal immunology in CRC staging". Mc Bain $\mathrm{AJ}$ et al [14] said that "Several hydrolytic and reductive bacterial enzymes ( $\beta$-glucuronidase, GN; $\beta$-glucosidase, GS; arylsulphatase, AS; azoreductase, AR; nitroreductase, NR) involved in production of mutagenic or genotoxic metabolites were measured in human colonic contents. Cell-associated AS and extracellular GS were approximately twice as high in the distal colon compared with the proximal bowel, while AR changed little throughout the gut. Measurements of these enzymes in faeces from seven healthy donors confirmed that the majority were cell-associated and demonstrated high levels of inter-individual variability. NR decreased four-fold between the proximal and distal colon while extracellular GN was reduced by $50 \%$. Most probable number (MPN) analysis on faeces obtained from six healthy donors showed that counts of intestinal bacteria producing GS and AR were c. 1010 and 1011/g, respectively, in all samples tested. Numbers of GN- and AS-forming organisms were between two and three orders of magnitude lower. Inter-individual carriage rates of bacterial populations synthesising NR were highly variable. Screening of 20 pure cultures of intestinal bacteria, belonging to six different genera, showed that Bacteroides ovatus, in particular, synthesized large amounts of GS, whereas B. fragilis, B. vulgatus and Bifido bacterium pseudolongum formed the highest cell-associated levels of GN. In general, bifido bacteria and Lactobacillus acidophilus did not produce significant amounts of AR. All five clostridia studied (Clostridium bifermentans, C. septicum, C. perfringens, C. sporogenes and C. butyricum) produced NR and AR, as did the bacteroides (B. fragilis, B. ovatus and B. vulgatus). Escherichia coli and C. perfringens formed large amounts of NR. Levels of AS production were invariably low and few of the organisms screened synthesized this enzyme. In-vitro studies investigating the effect of intestinal transit time on enzyme production, in a three-stage (V1-V3) continuous culture model of the colon operated at system retention times (R) of either 31.1 or 
68.4 h, showed that specific activities of GS were up to four-fold higher (V3) at $\mathrm{R}=31.1 \mathrm{~h}$. Bacteriological analysis demonstrated that representative populations of colonic micro-organisms were maintained in the fermentation system, and indicated that changes in GS activity were not related to numbers of the predominant anaerobic or facultative anaerobic species within the model, but were explainable on the basis of substrate-induced modulation of bacterial metabolism. more often in the hindgut than in the small intestine, indicating that the colonic microbiota plays an important role in carcinogenesis. It has also been suggested that a mechanism whereby intestinal bacteria may be involved in these processes is by the production of carcinogenic metabolites from non-toxic precursor molecules, and a variety of hydrolytic and reductive enzymes responsible for carcinogen production are known to be produced by colonic micro-organisms studies with rats have demonstrated that diet can have a significant effect on enzyme synthesis in intestinal bacteria. When animals fed a grain-based malware changed to a predominantly meat diet, they excreted significantly higher levels of faecal NR, AR and GN, while GS activities in faeces were reduced.

From these observations, it was suggested that the other environmental factors influence bacterial enzyme activities in the large intestine, for example, intracellular GN in E. coli and C. perfringens has been reported to be increased by bile salts ,whereas GN and bile acid excretion in human faeces are known to be elevated during consumption of high fat diets Enumeration of faecal micro-organisms forming hydrolytic and reductive enzymes involved in producing genotoxic substances, by MPN methods, demonstrated that the predominant colonic anaerobes formed AR. Moreover, these results showed great variability in the relative numbers of bacteria producing other enzymes associated with genotoxicity, particularly with respect to GN and NR. Although few data are available concerning the identities or physiologies of bacteria involved in NR formation in the large intestine, Rafii identified several clostridia that produced this enzyme, in a single human faecal sample, including C. leptum, C. paraputrijcum and C. clostridiiforme. Their investigations confirmed that aromatic amines were the primary products of NR activity, and that the enzymes involved had broad substrate specificities transit time of digestive material through the large intestine is an important factor affecting growth and metabolism of colonic bacteria, particularly in the carbohydrate-limited distal bowel, and is typically c.60-70 $\mathrm{h}$ in the United Kingdom". Povey AC et al [15] said that "The promutagenic lesion O(6)-methyldeoxyguanosine (O(6)-MedG), a marker of exposure to many N-nitroso compounds (NOC), can be detected in normal and tumor DNA isolated from colorectal tissue. The biological significance of this exposure is, as yet, unknown but in situ NOC formation is bacterially catalyzed suggesting that NOC formation and potentially DNA alkylation will vary throughout the large bowel.

To determine if O(6)-MedG levels in colorectal DNA vary within the large bowel. The study includes 62 men and women undergoing surgery for colorectal tumours in the north west of England. O(6)-
MedG levels were measured in paired normal and tumour DNA samples. DNA was digested to nucleosides, fractionated by HPLC, and purified $\mathrm{O}(6)$-MedG quantified by a radioimmunoassay(6)MedG was detected in 27 out of a total of 62 (43\%) normal DNA samples and in 30 of 58 (52\%) tumour DNA samples: it was present at concentrations of $<0$. 01-0.94 and $<0.01-0.151$ micromole $\mathrm{O}(6)-\mathrm{MedG} / \mathrm{mol}$ deoxy guanosine for normal and tumour DNA, respectively. Levels of O(6)-MedG in normal, but not tumour, DNA from the proximal colon were lower than those found in DNA from either the sigmoid colon $(p=0.03)$ or rectum $(p=0.05)$. When the analysis was restricted to samples that contained $0(6)-M e d G$, similar results were obtained in that $\mathrm{O}(6)-$ MedG levels in normal DNA were lower in the proximal colon than in the sigmoid colon $(p=0.04)$ or rectum $(p=0.03)$. DNA alkylation varied within the large bowel possibly due to in situ NOC formation and was highest in areas of the colon and rectum where the highest incidence of large bowel tumours occurs, suggesting that DNA alkylation may play a role in the etiology of colorectal cancer" [16]. Larsson SC et al said that "Although there is considerable evidence that high consumption of red meat may increase the risk of colorectal cancer, data by subsite within the colon are sparse. The objective of our study was to prospectively examine whether the association of red meat consumption with cancer risk varies by subsite within the large bowel. We analyzed data from the Swedish Mammography Cohort of 61,433 women aged 40-75 years and free from diagnosed cancer at baseline in 1987-1990. Diet was assessed at baseline using a self-administered food-frequency questionnaire. Over a mean follow-up of 13.9 years, we identified 234 proximal colon cancers, 155 distal colon cancers and 230 rectal cancers. We observed a significant positive association between red meat consumption and risk of distal colon cancer ( $p$ for trend $=0.001$ ) but not of cancers of the proximal colon ( $\mathrm{p}$ for trend $=0.95$ ) or rectum ( $\mathrm{p}$ for trend = 0.32). The multivariate rate ratio for women who consumed 94 or more g/day of red meat compared to those who consumed less than $50 \mathrm{~g} /$ day was 2.22 (95\% confidence interval [CI] 1.34-3.68) for distal colon, 1.03 (95\% CI 0.67-1.60) for proximal colon and 1.28 (95\% CI 0.83-1.98) for rectum. Although there was no association between consumption of fish and risk of cancer at any subsite, poultry consumption was weakly inversely related to risk of total colorectal cancer ( $\mathrm{p}$ for trend $=0.04$ ). These findings suggest that high consumption of red meat may substantially increase the risk of distal colon cancer. Future investigations on red meat and colorectal cancer risk should consider cancer subsites separately “. P Hachichmid et al. [18] said that " The pattern of colorectal polyps and carcinomas have been investigated in Southern Iran through prospective review of autopsy colons and a retrospective review of surgical pathology and autopsy specimens. It was found that the frequency of polyps was very low in our region (1.56\%). This was not improved by increasing the visibility using a magnifying lens. The frequency increased with age and males predominated. The polyps were most common in the colon and especially the ascending colon. Most, if not all, polyps were adenomatous in type. None of the surgical specimens over an 11-year period had adenomatous polyps of the colon and the only polyps noted were of 
the rectum. Thus, colonic polyps did not appear to be an important clinical problem in Southern Iran."

The frequency with which adenomatous polyps were seen in our region is one of the lowest recorded in the literature. Compared with Hawaiian-Japanese, the US population, and Australia or even Colombia, our polyp frequency is nearly nil. The data show that colonic carcinoma, though present in Fars, appears to be considerably lower in frequency as compared to the USAs represented by Connecticut. Increasing frequency with age was also noted. Rectal carcinomas were slightly more frequent than those of the colon. In the colon, carcinoma was more frequently found in the cecum and ascending colon. Nearly all carcinomas of the colon and rectum were adenocarcinomas, a similar finding to that in the West. In a recent review of epidemiologic findings of postulations have been reached:" There are low risk populations (socalled"endemic") where colon carcinomas predominate in the cecum and ascending colon. The maximum incidence in this group is in ages 50-55 with a female preponderance. Transition from an "endemic" to "epidemic"(high-risk) group may reflect introduction of a carcinogen and is initially noted by a rise in sigmoid cancers among men over 55. This is followed by a rise in female sigmoid cancers which is then followed by a rise in cecal and ascending colon cancer. In testing this model with the Fars data, we find that our frequency places us amongst the low-risk group. Cecal and ascending colon cancers predominate with nearly three out of five cancers being found in this region: in fact, about\% of the cancers were in the cecum. There was also a rise a maximum by age. On the other hand, there was an overall male predominance of both colon and rectal carcinomas. This was most visible after age ; before age there was a greater equality in frequency between males and females with an occasional female predominance. There did not appear to be an increase in male sigmoid cancers over age. Though the hypothesis requires testing overtime, the initial confirmation in our population is made difficult by the sex-related data; being a Moslem country with less female referral to hospitals and clinics may explain this difference in observation, if indeed it is true. With regard to the possible relationship between the food fiber content and the frequency of colorectal polyps-diverticula-carcinomas6*'our indigent population from which most of our necropsies are derived are known to consume high-fiber content food. Finally, it should be pointed out that though the relation between adenomatous polyps and adenocarcinoma is still controversial, our findings do confirm the observation that both these diseases are of low frequency in our population. Perhaps this parallelism in frequency along with age and sex prevalence should continue to open the possibility that adenomatous polyps and adenocarcinoma are somehow related in that adenomatous polyps may be precancerous or that both polyps and cancer may relate to a common though yet unidentified etiology. It should be noted, that in our series the large bowel segments with highest cancer frequency(i.e., cecum and rectum) did not harbor".

Noam Harpaz et al. [19] said that "Since the first published reports of an association between colorectal cancer (CRC) and the 2 major forms of idiopathic chronic inflammatory bowel disease(IBD), ulcerative colitis (UC) in 19251 and Crohn disease(CD) in 1948,2 CRC has become recognized as a leading cause of long-term mortality in men and women with IBD, most of whom become victims by middle age. For the past 3 decades, efforts at cancer prevention in this population have been based on an empirical strategy of scheduled colonoscopy surveillance examinations with biopsies to identify dysplasia, the earliest recognizable precursor of CRC and the most reliable marker of imminent cancer risk. A widely cited comprehensive metanalysis of 19 studies with agestratified data10 has estimated that the cumulative incidence of CRC in UC is $2 \%$ after 10 years, $8 \%$ after 20 years, and $18 \%$ after 30 years of disease. several more recent studies Case control and cohort studies have reported a greater than 2-fold increased risk of CRC in patients with IBD who have first-degree relatives with CRC and a 9-fold increased risk if the relatives had CRC before age 50 years. Inflammatory bowel disease-associated CRC occurs in a background of chronically inflamed mucosa, although direct endoscopic visualization, compared with microscopic assessment, often underestimates the intensity and extent of inflammation. Most studies9, have reported CRC cases in UC predominated in the left colon, especially the sigmoid colon and rectum, but others have reported a roughly even distribution of lesions on either side of the splenic flexure in both UC and CD. The frequency of multiple synchronous cancers in IBD is $10 \%$ to $30 \%$, especially in younger patients, and the simultaneous occurrence of 3 or more lesions is not unusual; by comparison, the frequency of 2 synchronous cancers in the general population is $3 \%$ to $5 \%$ and that of 3 or more is virtually nil. Dysplasia-Cancer Sequence Pathologists of the precolonoscopic era were the first to recognize dysplasia as a precursor of CRC in IBD and to propose periodic monitoring for dysplasia with rectal biopsies as a means of controlling cancer mortality Inflammatory bowel disease-associated dysplasia and CRC share common gene mutations and altered gene expression profiles.

\section{Dysplastic Versus Reactive Epithelium}

The distinction in endoscopic biopsy specimens between dysplasia and reactive epithelial changes elicited by colitis can be challenging, especially when there is a background of active or resolving inflammation. Nevertheless, the notion that a diagnosis of dysplasia may simply be disregarded when it occurs in the setting of active inflammation is baseless. One of the hallmarks of reactive crypts is a base-to surface epithelial maturation gradient in which phenotypically immature, mitotically active, basal colonocytes differentiate into mature surface cells featuring small, norm chromatic nuclei, distinct absorptive and goblet cell phenotypes, and absent mitoses. In the setting of active or resolving inflammation, the proportions of immature cells tend to expand and to displace the maturation gradient closer to the surface. Dysplastic epithelium, in contrast, extends uniformly along the crypt axis and surface epithelium with little or no surface maturation. Other features that favor dysplasia overreactive mucosa include diffuse nuclear hyperchromasia, macro nucleoli, atypical mitotic figures, loss of cellular polarity, and dirty intraluminal necrosis. Nondysplastic epithelium also tends to be more responsive to local variations in 
inflammatory activity than dysplastic epithelium. As a result, foci of cryptitis or erosions in the absence of dysplasia elicit maturation micro gradients, that is, transitions from regenerative to mature colonocytes within a single crypt or group of adjoining crypts.
Dysplastic epithelium usually manifests a blunted reaction to focal inflammation, although it may retain the ability to regenerate in the vicinity of ulcers [19] (Figure 4).

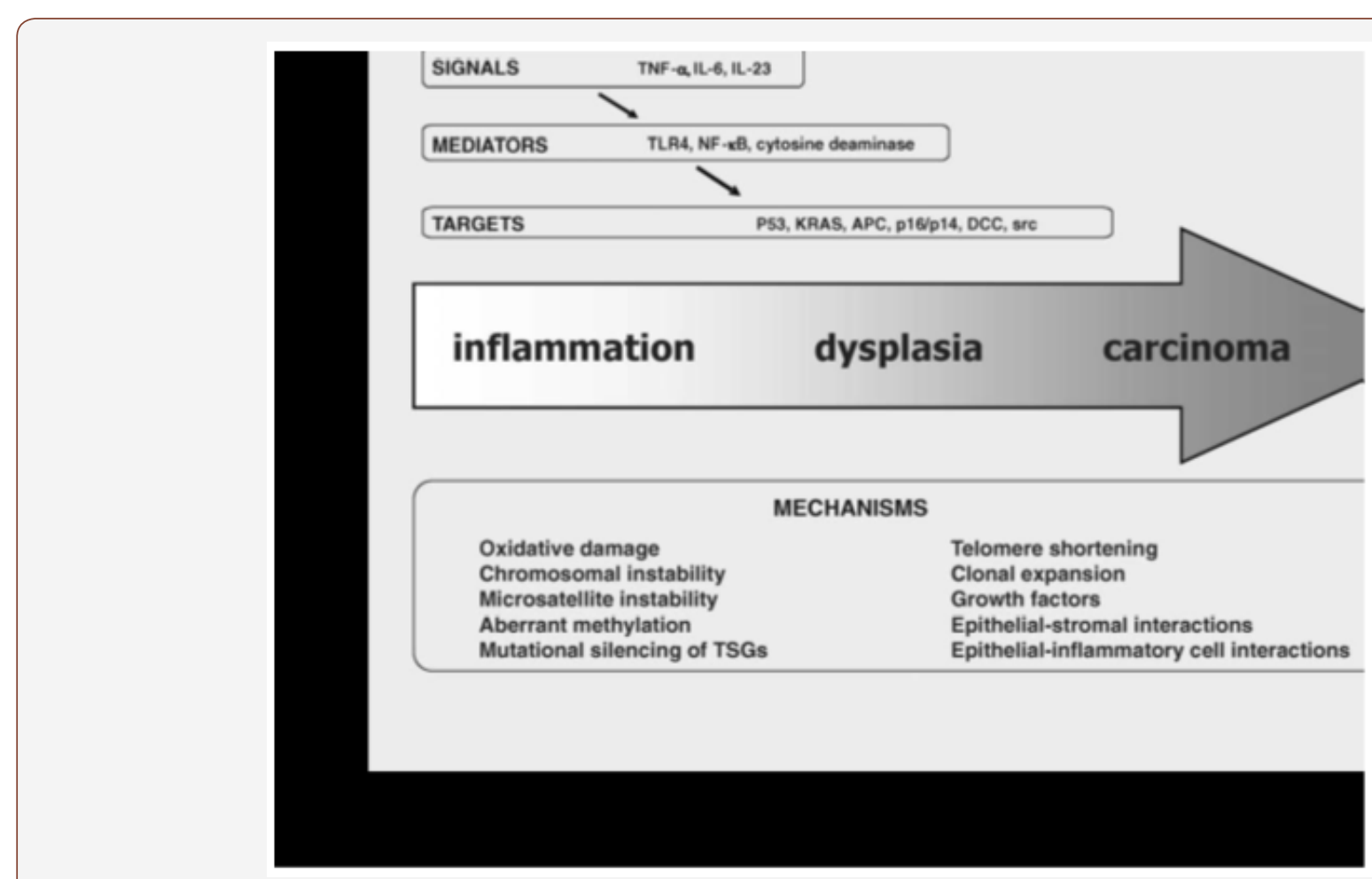

Figure 4: Molecular pathogenesis of colorectal dysplasia and cancer in inflammatory bowel disease. Summary of molecular signals, mediators, targets, and mechanisms implicated in progression from inflammation to cancer. Abbreviations: IL, interleukin; TLR, toll-like receptor; TNF, tumor necrosis factor; TSG, tumor suppressor gene [20].

Schneider A et al. [20]: "In a retrospective investigation, the clinical and pathomorphological findings of 27 patients (18 men, 9 women) with ulcerative colitis and colorectal carcinoma were analyzed. Their average age at the onset of the disease was 32.4 years, the duration of the illness at the time of the diagnosis of carcinoma 12.5 years, and age at the time carcinoma was diagnosed 45.4 years. In 23 patients (85 \%), total colitis presented. In 15\% of the cases, multiple carcinomas developed. Eighty-six percent of the carcinomas were located in the left colon, and $54 \%$ of all carcinomas were in the rectosigmoid. Forty-four percent of the carcinomas were primarily diffusely stenosing lesions. Nineteen percent were mucus-producing adenocarcinomas. Eighty-nine percent of the lesions were well or moderately well differentiated. At the time of the diagnosis of carcinoma, $35 \%$ of the patients had lymph node or distant metastasis. In $85 \%$ of the cases, dysplastic lesions were found in the close and more distant vicinity of the carcinoma". Riegler G et al. [21]: "To take a census of the cases of colorectal cancer in ulcerative colitis patients observed in Italy and to evaluate the clinical presentation of neoplastic complication. Experts from 28 Italian centers specialized in the management of inflammatory bowel disease or malignancies participated to the study. They were invited to send clinical data of patients with ulcerative colitis complicated by colorectal cancer or high-grade dysplasia consecutively observed between 1985 and 2000. One hundred and twelve patients (92 with cancer and 20 with high-grade dysplasia) were collected. Fourteen of them had undergone colectomy and ileorectal anastomosis for ulcerative colitis. Data of surgical patients were analyzed separately. The mean age at diagnosis of ulcerative colitis and colorectal cancer patients was 39.3 and 53.2 years, respectively, and the mean duration between diagnosis of ulcerative colitis and cancer was 13.9 years (range 0-53). Inflammation was proximal to the splenic flexure in 71 cases $(76.3 \%)$. One hundred and three colorectal cancers were registered (93 patients with single lesion and five patients with two synchronous cancers), with $76.7 \%$ of cancers being located in the left colon. As to the surgical patients, the mean age at diagnosis of ulcerative colitis and cancer was 28.9 and 47.0 years, respectively, and the mean diagnostic interval for ulcerative colitis and cancer was 18.1 years. Only 51 out of 112 patients were in follow-up. An early diagnosis of neoplasia (high grade dysplasia, stage A or B sec. Dukes) occurred in $72.5 \%$ of patients who were subjected to endoscopic surveillance and in $48.0 \%$ of patients who did not undergo endoscopic surveillance $(\mathrm{p}=0.02)$.These data show an earlier diagnosis of cancer in patients who had undergone endoscopic surveillance. The poor compliance to the follow-up program, reduces its effectiveness. Moreover, total colectomy allows an easier follow-up, with only the rectum being controlled. Colectomy with ileorectal anastomosis or proctocolectomy with ileo-anal anastomosis, could represent 
a valid alternative in patients at high risk of cancer who refuse endoscopic surveillance". Ewa Pasquereau-Kotula et al. [22]: "For more than 50 years, clinical studies have strongly linked the presence of Streptococcus bovis biotype I, renamed Streptococcus gallolyticus subsp. gallolyticus (Sgg), to CRC. The first direct demonstration of the etiological role of Sgg isolate TX20005 in promoting CRC development was provided very recently. But Sgg has also been shown to behave as a passenger bacterium benefiting from tumor metabolites and able to secrete a specific "bacteriocin" that can kill closely related gut commensals thus enabling a better colonization of murine colon in CRC-context. We thus conclude that Sgg is both a passenger and a cancer promoting bacterium. But in order to become a driver bacterium, Sgg first needs to colonize the colon and it does so only if pre-malignant conditions exist. So Sgg is not the principal cause of CRC but an auxiliary factor accelerating the development of CRC. Ultimately, the strong association of Sgg with CRC constitutes a solid argument to recommend a systematic colonoscopy for assessment of occult neoplasia in patients suffering of Sgg infections. Development of new molecular tools for the sensitive and specific detection of specific CRC-associated bacteria should help in the early detection of subclinical colonic lesions but may also add a weapon in the oncologists' arsenal as demonstrated recently. A future area of investigation will be to study the relationship between Sgg and host immunity, another important player in CRC development". Ritesh Kumar et al. [23]: "Streptococcus gallolyticus subsp. gallolyticus (Sg) has long been known to have a strong association with colorectal cancer (CRC). This knowledge has important clinical implications, and yet little is known about the role of Sg in the development of CRC. Here we demonstrate that Sg promotes human colon cancer cell proliferation in a manner that depends on cell context, bacterial growth phase and direct contact between bacteria and colon cancer cells. In addition, we observed increased level of $\beta$-catenin, c-Myc and PCNA in colon cancer cells following incubation with Sg. Knockdown or inhibition of $\beta$-catenin abolished the effect of Sg. mice administered with Sg had significantly more tumors, higher tumor burden and dysplasia grade, and increased cell proliferation and $\beta$-catenin staining in colonic crypts compared to mice receiving control bacteria. Finally, we showed that Sg is present in the majority of CRC patients and is preferentially associated with tumor compared to normal tissues obtained from CRC patients. These results taken together establish for the first time a tumor-promoting role of Sg that involves specific bacterial and host factors and have important clinical implications "[23].

Macfarlane GT et al. [24]: "Colonic contents were obtained from two human sudden-death victims within $3 \mathrm{~h}$ of death. One of the subjects (1) was methanogenic, the other (2) was a non-CH4 producer. Measurements of bacterial fermentation products showed that in both individuals short-chain fatty acids, lactate and ethanol concentrations were highest in the caecum and ascending colon. In contrast, products of protein fermentation, such as ammonia, branched chain fatty acids and phenolic compounds, progressively increased from the right to the left colon, as did the $\mathrm{pH}$ of gut contents. In Subject 1, cell population densities of methanogenic bacteria (MB) increased distally through the gut and methanogenic activity was lower in the right (0.78-1.18 mumol CH4 produced/h/g dry wt. contents) than in the left colon (1.34 mumol CH4 produced/ $\mathrm{h} / \mathrm{g}$ dry wt contents). Methane production rates did not correlate with MB numbers. Sulphate-reducing bacteria (SRB) were not found and dissimilatory sulphate reduction was not detected in any region of the colon. Methanogenic bacteria did not occur in subject 2 , but high numbers of SRB were present throughout the gut (ca $10(9) / g$ dry wt contents). Sulphate reduction rates were maximal in the ascending and transverse colons $(0.24$ and 0.22 mumol 35SO4(2-) reduced/h/g dry wt contents, respectively). Shortchain fatty acid production by caecal contents was up to eight-fold higher than contents from the sigmoid/rectum. These findings demonstrate significant differences in fermentation reactions in different regions of the large gut "[24]. Jianhong Peng et al. [25]: "Growing evidence has suggested that right-sided colon cancer (RCC) and left-sided colon cancer (LCC) should be considered as different tumor entities. stage III colon cancer is currently treated as the same entity with uniform therapy. This study was aimed at investigating the prognostic influence of tumor location in patients with stage III colon cancer receiving adjuvant chemotherapy after curative resection. This study demonstrated that tumors arising on different sides generate different prognostic outcomes in patients with stage III colon cancer who received adjuvant chemotherapy after curative resection. The full course of adjuvant chemotherapy might only benefit RCC patients. The tumor location is suggested to be an important clinical factor that can optimize the therapeutic adjuvant chemotherapy strategies. Further prospective studies are warranted to confirm these findings [25]. Mohamed E Salem et al. [26]: "Tumor sidedness has emerged as an important prognostic and predictive factor in the treatment of colorectal cancer. Recent studies demonstrate that patients with advanced right-sided colon cancers have a worse prognosis than those with left-sided colon or rectal cancers, and these patient subgroups respond differently to biological therapies. Historically, management of patients with metastatic colon and rectal cancers has been similar, and colon and rectal cancer patients have been grouped together in large clinical trials. Clearly, the differences in molecular biology among rightsided colon, left-sided colon, and rectal cancers should be further studied in order to account for disparities in clinical outcomes. We profiled 10,570 colorectal tumors (of which 2,413 were identified as arising from the left colon, right colon, or rectum) using nextgeneration sequencing, immunohistochemistry, chromogenic in-situ hybridization, and fragment analysis. Right-sided colon cancers had higher rates of microsatellite instability, more frequent aberrant activation of the EGFR pathway including higher BRAF and PIK3CA mutation rates, and increased mutational burden compared to left-sided colon and rectal cancers. Rectal cancers had higher rates of TOPO1 expression and Her2/neu amplification compared to both left- and right-sided colon cancers. Molecular variations among right-sided colon, left-sided colon, and rectal tumors may contribute to differences in clinical behavior. The site of tumor origin (left colon, right colon, or rectum) should certainly

Citation: Mauro Luisetto, Behzad Nili Ahmadabadi, Hossein Nili-Ahmadabadi, Ahmed Yesvi Rafa, Gamal Abdul Hamid, et al. Comparison of Risk Factors and Molecular Analysis of Right-Sided Colon and Left Sided Colon Cancer. Adv Can Res \& Clinical Imag. 2(2): 2019. ACRCI. 
be considered when selecting treatment regimens and stratifying patients for future clinical trials. These findings raise important questions: are right- and left-sided colon cancers biologically distinct from rectal cancers, and could this impact the choice of therapy for these distinct cancer groups ? Mutational analyses of primary tumors have suggested important differences in oncogenic driver mutations between colon and rectal cancers. In one study of 158 patients with metastatic colon cancers and 64 patients with metastatic rectal cancers, colon cancers had a higher BRAF mutation rate compared to rectal cancers $(13.3 \%$ vs. $3.1 \%, \mathrm{P}$ $<0.05)$, but lower rates of NRAS (0.6\% vs. $12.5 \%, \mathrm{P}<0.05)$ and TP53 mutations $(17.7 \%$ vs. $29.7 \%, \mathrm{P}<0.05)$. KRAS mutation rates were similar (37.3\% vs. $34.4 \%$; $P>0.05$ ) [9]. These differences offer insight into the potential use of EGFR inhibitors due to the fact that only patients with RAS and BRAF wild-type tumors derive benefit (although results from the study by Venook and colleagues suggested that cetuximab might actually be detrimental to patients with right-sided tumors, regardless of RAS status, meaning that other factors are coming into play).

Another study of 1,443 colon and rectal cancers demonstrated that a greater percentage (19-37\%) of proximal colon cancers are MMRd compared to only $1.6 \%$ of rectal cancers, suggesting that rectal cancers may be less responsive to novel immunotherapies such as anti-PD-1 or anti-PD-L1 (checkpoint) inhibitors. Using a large cohort of colorectal tumors submitted for profiling, we performed a systematic comparison of molecular alterations between tumors collected from different locations, including a large cohort of 1,445 rectal tumors. This sample size is in sharp contrast to the previous work done by The Cancer Genome Atlas (TCGA) which compared only 132 colon and 62 rectal tumors, although the TCGA data was obtained prospectively. Despite the exclusion of a large number of colon tumors from profiling analyses, specifically those without clear annotation of tumor location $(\mathrm{N}=8,041)$ and those taken from the transverse colon ( $\mathrm{N}=116)$, ours is still one of the largest cohorts analyzed and reported to date. All tumors were micro dissected and only tumor tissue was sampled for NGS, eliminating potential confounding mutations from non-malignant stromal regions that could influence genetic profiles by inappropriately suggesting epithelial-mesenchymal transition. Our biomarker analysis included only primary tumors due to the significantly different molecular profiles of primary and metastatic CRC. In this analysis, rectal tumors were more common in men whereas rightsided tumors were more common in women. This finding agrees with those from previous studies demonstrating that women are more likely to have right-sided tumors. The tendency of women to develop right-sided tumors likely reflects the protective role of estrogen in the development of CRC due to its anti-inflammatory effect on the tumor microenvironment. In fact, estrogen receptor expression is higher in right-sided than left-sided colon tumors. The rightward shift toward more proximal colon MMRd tumors in older female patients may be explained by the inevitable reduction in estrogen levels with age. We show a dramatic decrease in MMRd tumors when moving from right to left colon to rectum. MSI is caused by loss of MMR function and is generally found in about $15 \%$ of all CRCs, including around 3\% of CRCs associated with hereditary non-polyposis CRC (HNPCC), also known as Lynch Syndrome. Regardless of origin, patients with MMRd tumors are resistant to conventional chemotherapies, including 5-FU. these tumors carry a hypermutated phenotype associated with the generation of neoantigens, making them promising candidates for immune checkpoint inhibition. Our results show that approximately one quarter of right-sided colon tumors are MMRd and may benefit from treatment with checkpoint inhibitors, providing a very promising therapeutic opportunity for this subgroup of colon tumors with poor prognosis and limited treatment options. Others have observed that even in MMRp tumors, higher mutational load positively correlates with lymphocyte infiltration and improved CRC-specific survival. This study shows a significant decrease in the BRAF mutation rate from right colon (25\%) to left colon $(15 \%)$ to rectum $(3 \%)$. These values contrast significantly with the previously reported 5-10\% BRAF mutation rate, probably due to the fact that colon (both sides) and rectal tumors were grouped together in previous studies. Our findings confirm the results of previous studies demonstrating the right-sided predominance of BRAF-mutated CRC (comprising 22\% of right-sided, $4 \%$ of leftsided, and $2 \%$ of rectal CRCs). Mutated BRAF has been associated with MSI and CIMP. Patients with BRAF-mutated CRCs carry the worst overall prognosis among CRC patients and are notoriously refractory to therapy, despite best efforts with currently available BRAF-targeted agents and various combination therapies. One analysis of three independent cohorts that grouped rectal tumors with left-sided colon tumors showed that, although BRAF mutations were more prevalent in right-sided tumors, right-sided location was a negative prognostic variable independent of BRAF mutational status. Patients with RAS and BRAF wild-type rightsided tumors from the PRIME, PEAK, and 181 studies had inferior OS, progression-free survival (PFS), and objective response rate (ORR), and similar survival outcomes were observed in subgroup analyses of RAS wild-type tumors from the FIRE-3, CRYSTAL, and CALBG/SWOG 80405 studies. Our observations suggest that within the left-sided group, there is a decrease of BRAF mutation rate from left colon to rectum and that the different BRAF mutation rates might contribute to the different behavior of left- and right-sided colon cancers and rectal cancers. Additional important findings from our study include the significantly lower activation of the $\mathrm{PI} 3 \mathrm{~K} / \mathrm{AKT} / \mathrm{mTOR}$ pathway in rectal tumors compared to left- and right-sided colon tumors, with lower mutation rates of PIK3CA and PTEN in rectal tumors. Although agents that directly target the $\mathrm{PI} 3 \mathrm{~K} / \mathrm{AKT} / \mathrm{mTOR}$ pathway have been shown to be ineffective in CRC , PIK3CA mutations have been implicated in EGFR-targeted therapy resistance, as well as increased benefit from aspirin treatment. Loss of PTEN expression may also confer resistance to EGFRtargeted therapy. The higher rate of activating PIK3CA mutations and PTEN loss in left- and right-sided colon cancers compared to rectal cancers could contribute to a lower response to anti-EGFR therapies in some colon cancers and supports the use of alternative treatments. Epigenetic modifications that confer sensitivity to EGFR-targeted therapy (e.g., demethylation of the EREG promoter) 
occur more commonly in rectal tumors, but such epigenetic modifications were not assessed in our study. Additionally, the insulin-like growth factor (IGF) pathway has also been implicated in CRC oncogenesis but was not analyzed in our study. In a study of 69 patients with KRAS exon 2 mutant CRC, IGF-1 expression was higher in rectal than colon tumors, approaching statistical significance $(\mathrm{P}=0.06)$ and suggesting that rectal cancers harbor oncogenic pathways that are different from colon cancers. These are certainly markers to consider for future confirmatory analyses. Rectal cancers were shown to have higher expression of TOPO1 than colon cancers, which could be useful in the direction of irinotecan treatment-first demonstrated in the UK MRC FOCUS trial, but with limited validation from other trials. In the MAVERICC study, which compared first-line treatment using mFOLFOX6/bevacizumab with FOLFIRI/bevacizumab in patients with mCRC, those with left-sided mCRC attained longer median PFS with FOLFIRI/bevacizumab than mFOLFOX6/bevacizumab (13.8 vs. 10.2 months, $\mathrm{P}=0.040$ ); this was not seen in patients with right-sided metastatic colon cancer. Although this difference could be attributed to higher TOPO1 expression in the rectal tumors that were included in the left-sided cohort, further investigation is needed.

Xenograft model studies and the multicenter phase II HERACLES trial indicate that the Her2/neu pathway is an important therapeutic target in CRCs. Our current study suggests a trend toward higher prevalence of Her2/neu overexpression and amplification in rectal cancers compared to colon cancers, despite the low levels observed overall. The rate of Her $2 /$ neu overexpression in rectal cancer has previously been reported to be as high as $26.7 \%$. This finding could be helpful in the selection of patients for future clinical trials of Her2/neu-targeted therapies. Limitations of our study include lack of DNA methylation evaluation, CMS classification, and the restriction to molecular diagnostic tests widely used in the clinical setting (IHC, ISH, and NGS). In addition, primary or metastatic tumor specimens were categorized according to site of procurement, with no patient identification. Therefore, it is possible that the primary cohort may contain samples from primary sites in patients who also had metastatic tumors samples analyzed. Many tumors were excluded because their primary site was unknown $(\mathrm{N}=8,563)$, significantly limiting the power of this study to detect novel CRC mutations. Metastatic tumors (especially from the colon) were underrepresented relative to primary tumors, and patients with earlier stage colon cancer were also likely underrepresented in this study. This is because the stage at diagnosis was not reported and tumors are most commonly profiled at a later stage. Prior work by El-Deiry and colleagues demonstrated clear molecular differences among different metastatic sites of CRC and primary tumors, including higher Her2/neu expression in lung metastases than primary tumors ( $4 \%$ vs. $1.8 \%, \mathrm{P}=0.028$ ), higher rates of KRAS mutations in brain and lung metastases than other sites (65\% vs. $59 \%$ vs. $47 \%, \mathrm{P}=0.07$ and $<0.01$, respectively), and higher TOPO1 expression in metastatic tumors than primary tumors $(52 \%$ vs. $30 \%$ ) [16]. Rectal tumor specimens also may have received neoadjuvant chemoradiation, potentially altering their IHC profile. In one study of 225 resected rectal cancer specimens, Her2/neu amplification was seen in $45 \%$ of untreated tumors but only in $23 \%$ of tumors exposed to neoadjuvant chemoradiation ( $\mathrm{P}=0.009$ ). Finally, our analysis of tumor mutational load is exploratory given the small cohort of tumors assessed $(\mathrm{N}=70)$. Tumor sidedness has been shown to have a profound influence on clinical outcomes and the current study demonstrates, not only that left- and right-sided colon cancers differ in tumor biology, but also that rectal cancer have a tumor biology that is distinct from colon cancers. it appears that CRCs carry a continuum of molecular alterations from right to left, rather than having a sharp, clear-cut distinction" [26] (Figure 5).

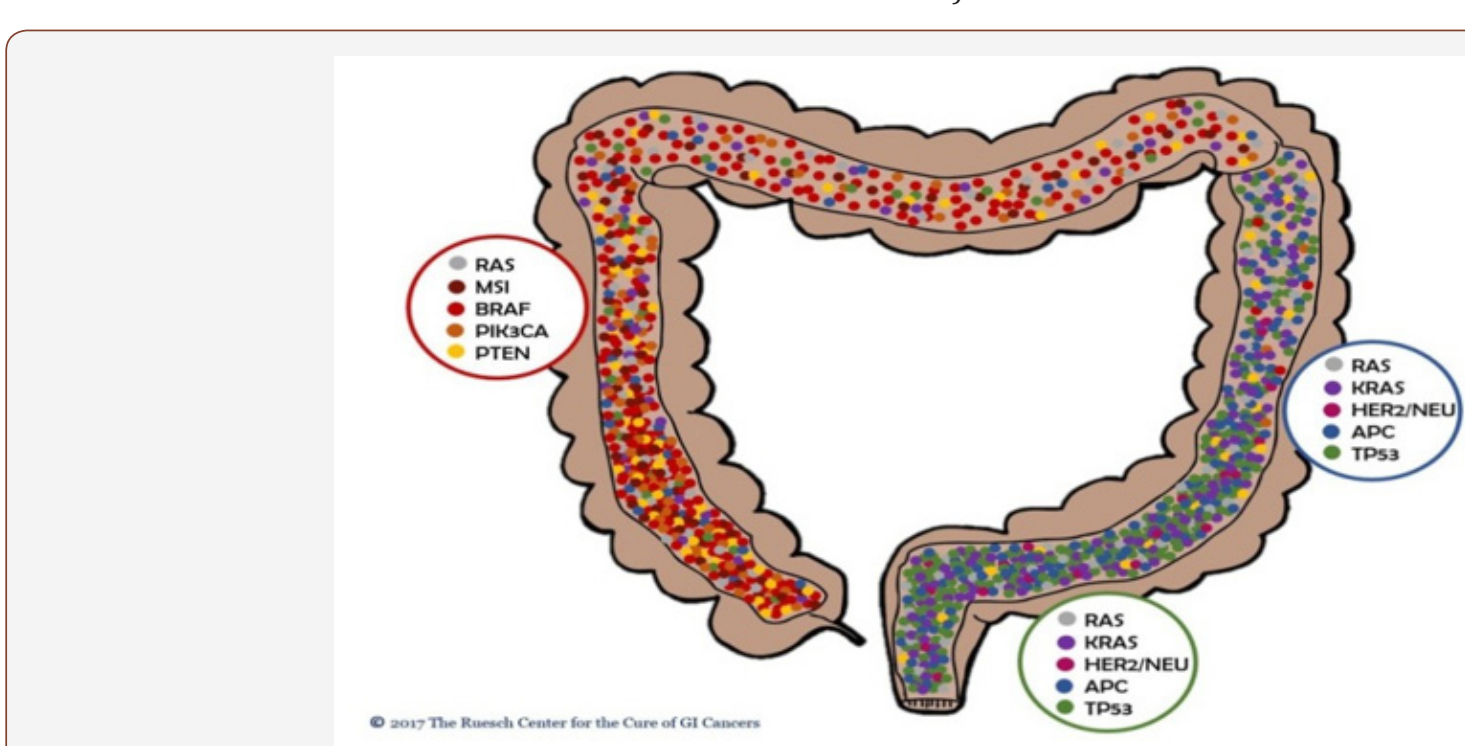

Figure 5: CRCs carry a continuum of molecular alterations from right to left, rather than having a sharp, clear-cut distinction [27]

Dae Ro Lim et al. [27]: "There are embryological origins, anatomical, histological, genetic, and immunological differences between right-sided colon cancer (RCC) and left-sided colon cancer (LCC). Many studies have sought to determine the survival and prognosis according to tumor location. This study aimed to analyze outcomes between RCC and LCC. Between January 2000

Citation: Mauro Luisetto, Behzad Nili Ahmadabadi, Hossein Nili-Ahmadabadi, Ahmed Yesvi Rafa, Gamal Abdul Hamid, et al. Comparison of Risk Factors and Molecular Analysis of Right-Sided Colon and Left Sided Colon Cancer. Adv Can Res \& Clinical Imag. 2(2): 2019. ACRCI 
and December 2012, data on 414 patients who underwent curative resection for RCC and LCC were retrieved from a retrospective database. Propensity score matching (1:1) was performed and RCC was identified in 207 and LCC in 207 patients. On average, RCC exhibited a more advanced $\mathrm{N}$ stage, increased tumor size, more frequently poorly differentiated tumors, more harvested lymph nodes, and more positivity of lymph vascular invasion than LCC. With a median follow-up of 66.7 months, the 5-year overall survival (OS) rates for RCC and LCC were $82.1 \%$ and $88.7 \%$, respectively, $(\mathrm{P}<.05)$. The 5-year disease-free survival (DFS) rates were $81.4 \%$ (RCC) and $88.3 \%$ (LCC; $\mathrm{P}<.05)$. In stage III cancers, the DFS rates were $61.1 \%$ (RCC) and $81.9 \%$ (LCC; $\mathrm{P}<.05$ ), while the OS rates were $65.6 \%$ (RCC) and 78.6\% (LCC; $\mathrm{P}=.056$ ). On the basis of present data, LCC exhibited better survival outcomes than RCC after curative resection. Especially in stage III, LCC showed better oncologic outcomes. Proper specialized treatment related to the location of colon cancer is needed". Miao-Zhen Qiu et al. [28]: "Mountain of studies has showed that right-sided colon cancer (RSCC) and leftsided colon cancer (LSCC) have different clinical presentation and biologic features and should be considered as two distinct disease entities. The survival difference between RSCC and LSCC remains controversial. Using Surveillance, Epidemiology, and End Results (SEER) database, we identified colon adenocarcinoma patients from 2004 to 2013. The 5-year cause-specific survival (CSS) was our primary endpoint. All statistical analyses were performed using the Intercooled Stata 13.0. All statistical tests were two-sided. The study included 95,847 (58.72\%) RSCC and 67,385 (41.28\%) LSCC patients. RSCC patients were older, more often females, more Caucasian, more unmarried, more advanced $\mathrm{T}$ and $\mathrm{N}$ stage, larger tumor sizes, and more poorly differentiated tumor, while LSCC patients had more stage IV diseases. Location was an independent prognostic factor in the multivariable analysis. Compared with RSCC patients, the hazard ratio for LSCC was 0.87, 95\% CI: $0.85-$ $0.89 \mathrm{P}<0.001$. There was no survival difference between RSCC and LSCC in the following situations: older than 68 years old, T3-4, N0, poorly differentiated, and undifferentiated diseases. We firstly reported that RSCC patients had a better prognosis than LSCC in mucinous adenocarcinoma/signet ring cell carcinoma patients.
RSCC patients also had a better prognosis than LSCC in stage II disease. There is a need for further subdivisions when analyzing the survival difference between RSCC and LSCC patients. RSCC had lower mortality rate than LSCC in stage II disease and mucinous adenocarcinoma/signet ring cell carcinoma patients". Yaqi Wang et al. [29]: "To investigate whether young patients exhibit different characteristics and survival according to tumor location and stage using data from the Surveillance, Epidemiology, and End Results (SEER) database. Young patients (20-49 years old) with stage I-III colon cancers were identified from the SEER program from 1990 to 2014. Kaplan-Meier survival analysis and Cox proportional hazards regression were used to analyze the data. Subset analyses were also done among different age and stage subgroups. Of 8197 patients, 3709 (45.2\%) had right-sided colon cancers (RCCs). Patients with RCCs were more likely to be male, to be younger, and to have more poorly differentiated and more advanced tumors. The Kaplan-Meier survival curves and univariate survival models revealed that left-sided colon cancers (LCCs) had lower mortality for all stages combined and stage III, but higher mortality for stage II, compared with right-sided tumors. multivariate Cox regression models showed no significant survival differences by location for all patients (adjusted hazard ratio [HR], 0.95; 95\% confidence interval [CI], 0.86-1.05; $\mathrm{P}=0.34$ ) or for stage I (adjusted HR, 1.47; 95\% CI, 0.82-2.63; $\mathrm{P}=0.20$ ). Stage II left-sided cancers had higher mortality (adjusted HR, 1.24; 95\% CI, 1.00-1.54; P=0.048), whereas stage III left-sided cancers had lower mortality (adjusted $\mathrm{HR}, 0.86$; 95\% CI, 0.77-0.97; $\mathrm{P}=0.01$ ). For 20- to 39-year-old patients, a significant difference was only found in stage II disease, with a higher mortality for left-sided tumors (adjusted HR, 1.82; 95\% CI, 1.12-2.97; $\mathrm{P}=0.02$ ). for 40 - to 49-year-old patients, a significant difference was only found in stage III disease, with a lower mortality for left-sided tumors (adjusted HR, 0.83; 95\% CI, 0.72-0.95; $\mathrm{P}=0.008)$. In patients younger than 50 years, there were no significant differences in mortality between RCCs and LCCs for all stages combined after adjusting for multiple clinicopathological features. RCCs had lower mortality in stage II (especially in 20- to 39-year-old patients) and higher mortality in stage III (especially in 40- to 49-year-old patients)".

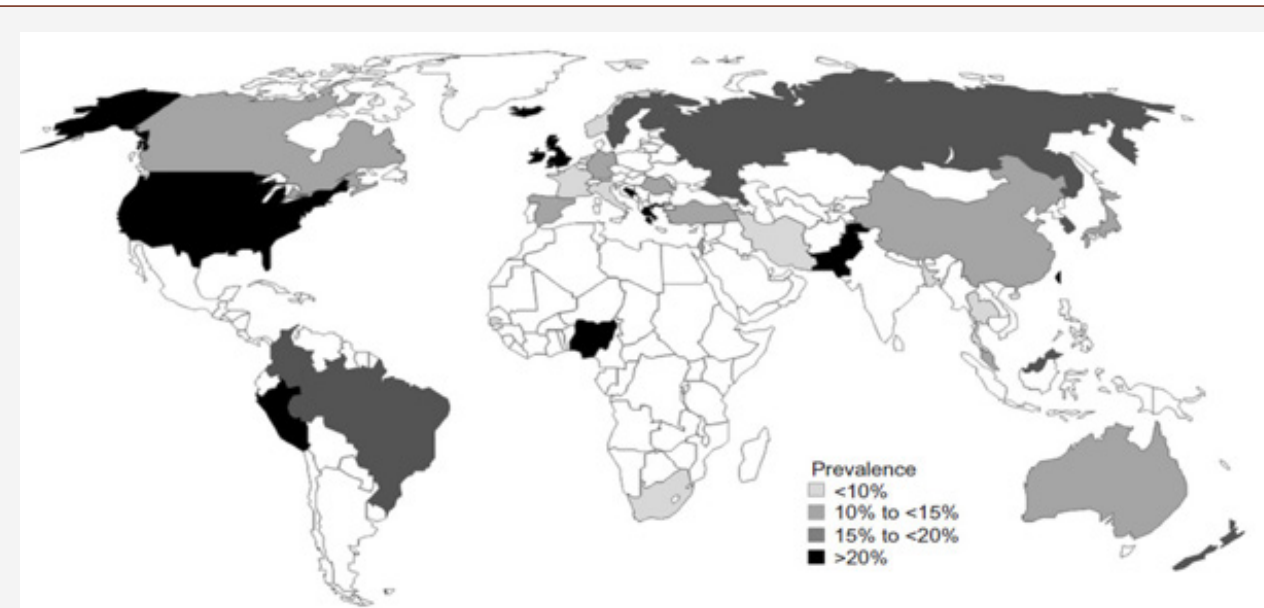

Figure 6: Worldwide prevalence of irritable bowel syndrome [53]. 
Elisa Tiratterra et al. [30]: "Bile acids (BAs) are the end product of cholesterol catabolism. Their synthesis is regulated by the nuclear receptor farnesoid $\mathrm{X}$ receptor, also involved in the control of their enterohepatic circulation. Inflammatory bowel diseases (IBD), which include Crohn's disease (CD) and ulcerative colitis (UC), are multifactorial diseases characterized by diarrhea. The pathogenesis of diarrhea in IBD is still debated. The most important factor is the inflammatory process of the intestinal wall, causing alterations of solute and water absorption/secretion, deterioration of epithelial cell integrity, disruption of the intestinal microflora homeostasis, and impairment of specific transport mechanisms within the gut (including that of BAs). In this review, we summarize the current state of the art in this area and we critically evaluate the alterations of BA metabolism in patients with CD and UC. Bile acids (BAs) are the end product of cholesterol catabolism; they contribute to intestinal nutrient absorption and the biliary transport of lipids, toxic metabolites, and xenobiotics. Their synthesis is regulated by the farnesoid $\mathrm{X}$ receptor (FXR), also involved in the control of their enterohepatic circulation. Inflammatory bowel disease (IBD) is a term that encompasses multifactorial diseases characterized by various symptoms, among which diarrhea represents an important target for treatment. The pathogenic role of BAs in chronic IBD has long been debated. BA malabsorption (BAM) plays a major role in diarrhea, because luminal BAs result in colonic secretion of water and electrolytes and the induction of propagated contractions. Inflammatory processes happening in the intestinal wall are the major contributing factors to IBD; nevertheless, impaired metabolism of BAs and BA-FXR interaction are involved in the pathophysiology of this disease. We know that defective BA metabolism can be related to many causes, such as impaired biosynthesis from cholesterol, faulty transport of hepatocytes or enterocytes through the cellular membrane, defective transport among the physiological compartments involved in the enterohepatic circulation, or abnormal bacterial overgrowth in the large intestine. Under these conditions, administration of BA sequestrants, which can reduce the colonic concentration of bile salts, leads to symptomatic improvements in diarrhea.

Several analytical protocols are currently used for the diagnosis of diarrhea related to BAs, including75selenium homotaurocholic acid test retention, serum $\mathrm{C} 4$, fibroblast growth factor (FGF)19 , and fecal BA measurements. Unfortunately, these methods do not always provide consistent results and conflicting reports may be found in the literature. This review aims to summarize what is currently known about the pathogenic role of BAs and BA metabolism in IBD, providing a general overview of BA physiology and perturbations in their metabolic homeostasis, and to develop a useful tool that may be applied in clinical practice". Nuri Faruk Aykan [31]: "Colorectal cancer (CRC) is the third most common cancer in men and the second in women worldwide. More than half of cases occur in more developed countries. The consumption of red meat (beef, pork, lamb, veal, mutton) is high in developed countries and accumulated evidence until today demonstrated a convincing association between the intake of red meat and especially processed meat and CRC risk. In this review, metaanalyses of prospective epidemiological studies addressed to this association, observed link of some subtypes of red meat with CRC risk, potential carcinogenic compounds, their mechanisms and actual recommendations of international guidelines are presented. As a conclusion, accumulated evidence of prospective epidemiological studies and their meta-analyses shows that red meat and processed meat convincingly increases CRC risk by 20$30 \%$. Regarding specific red meat subtypes, the association with increased risk was found for beef consumption in two trials (one of them is weakly associated), for pork consumption in three trials and for lamb intake in one trial. An interesting observation is the existence of this risk only for pork intake and rectum cancer and lamb intake and colon cancer, respectively in one trial. Beef and pork consumption were found also to be associated with colon cancer only in women, in one trial.

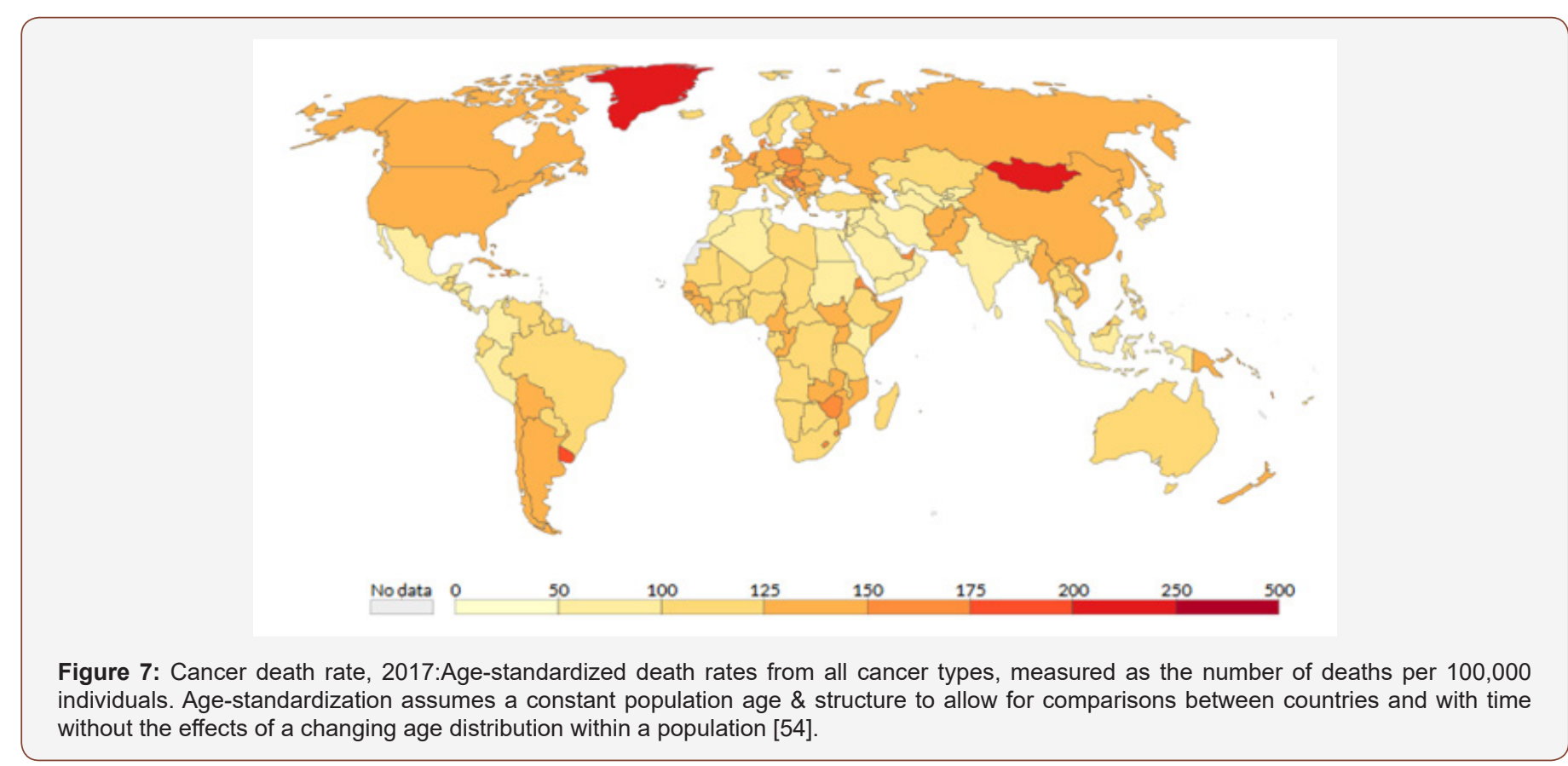

Citation: Mauro Luisetto, Behzad Nili Ahmadabadi, Hossein Nili-Ahmadabadi, Ahmed Yesvi Rafa, Gamal Abdul Hamid, et al. Comparison of Risk Factors and Molecular Analysis of Right-Sided Colon and Left Sided Colon Cancer. Adv Can Res \& Clinical Imag. 2(2): 2019. ACRCI. 
Whether CRC is one disease or the existence of 2 categories of CRC (colon and rectum or proximal and distal or right and left colon) and the link between etiologic factors and molecular subtypes are another hot topic of discussion, which need further investigations"[31]. According to guidelines today,51-55 recommended amount of red meat for healthy people is 500 $\mathrm{g} /$ week or $70 \mathrm{~g} /$ day. They recommend also limiting intake of processed meat. White meat (fish and poultry) is not associated with CRC risk and is recommended safely. To diminish carcinogenic effects of HCAs, diet should be high in dietary fibre sources such as wheat bran and vegetables. Formation of HCAs can be reduced by avoidance of exposure of meat surfaces to flames, usage of aluminum foil to wrap meat before oven roasting and microwave cooking “. Teresa Norat et al. [32]: "Current evidence suggests that high red meat intake is associated with increased colorectal cancer risk. High fish intake may be associated with a decreased risk, but the existing evidence is less convincing. We prospectively followed 478040 men and women from 10 European countries who were free of cancer at enrollment between 1992 and 1998. Information on diet and lifestyle was collected at baseline. After a mean follow-up of 4.8 years, 1329 incident colorectal cancers were documented. We examined the relationship between intakes of red and processed meat, poultry, and fish and colorectal cancer risk using a proportional hazards model adjusted for age, sex, energy (nonfat and fat sources), height, weight, work-related physical activity, smoking status, dietary fiber and folate, and alcohol consumption, stratified by center. A calibration sub study based on 36994 subjects was used to correct hazard ratios (HRs) and 95\% confidence intervals (CIs) for diet measurement errors. All statistical tests were two-sided. Colorectal cancer risk was positively associated with intake of red and processed meat (highest $[>160 \mathrm{~g} /$ day $]$ versus lowest $[<20 \mathrm{~g} /$ day $]$ intake, $\mathrm{HR}=1.35$, $95 \% \mathrm{CI}=0.96$ to 1.88 ; Ptrend $=.03$ ) and inversely associated with intake of fish $(>80 \mathrm{~g} /$ day versus $<10 \mathrm{~g} /$ day, $\mathrm{HR}=0.69,95 \% \mathrm{CI}=$ 0.54 to 0.88 ; Ptrend<.001), but was not related to poultry intake. Correcting for measurement error strengthened the associations between colorectal cancer and red and processed meat intake (per $100-\mathrm{g}$ increase $\mathrm{HR}=1.25,95 \% \mathrm{CI}=1.09$ to 1.41 , Ptrend $=.001$ and $\mathrm{HR}=1.55,95 \% \mathrm{CI}=1.19$ to 2.02 , Ptrend $=.001$ before and after calibration, respectively) and for fish (per $100 \mathrm{~g}$ increase $\mathrm{HR}=0.70$, $95 \% \mathrm{CI}=0.57$ to 0.87 , Ptrend $<.001$ and $\mathrm{HR}=0.46,95 \% \mathrm{CI}=0.27$ to 0.77 , Ptrend=.003; before and after correction, respectively). In this study population, the absolute risk of development of colorectal cancer within 10 years for a study subject aged 50 years was $1.71 \%$ for the highest category of red meat intake and $1.28 \%$ for the lowest category of intake and was $1.86 \%$ for subjects in the lowest category of fish intake and $1.28 \%$ for subjects in the highest category of fish intake ". Glebov OK et al. [17]: "Distinct epidemiological and clinic-pathological characteristics of colorectal carcinomas (CRCs) based on their anatomical location suggest different risk factors and pathways of transformation associated with proximal and distal colon carcinogenesis. These differences may reflect distinct biological characteristics of proximal and distal colonic mucosa, acquired in embryonic or postnatal development, that determine a differential response to uniformly distributed environmental factors. Alternatively, the differences in the epidemiology of proximal and distal CRCs could result from the presence of different pro-carcinogenic factors in the ascending versus descending colon, acting on cells with either similar or distinct biological characteristics. We applied cDNA microarray technology to explore the possibility that mucosal epithelium from adult proximal and distal colon can be distinguished by their pattern of gene expression. In addition, gene expression was studied in fetal (17-24 weeks gestation) proximal and distal colon. More than 1000 genes were expressed differentially in adult ascending versus descending colon, with 165 genes showing >2-fold and 49 genes showing $>3$-fold differences in expression. With almost complete concordance, biopsies of adult colonic epithelium can be correctly classified as proximal or distal by gene expression profile. Only 87 genes were expressed differently in ascending and descending fetal colon, indicating that, although anatomically relevant differences are already established in embryonic colon, additional changes in gene expression occur in postnatal development".

\begin{tabular}{|c|c|c|c|c|}
\hline Risk Factor & Overall CRC Risk & Proximal CRC Risk & Distal CRC Risk & Rectal Cancer Risk \\
\hline Physical Activity & $y$ & & & \\
\hline Height & 수 & 7 & & \\
\hline Body Mass Index & (male) & (male) & (male) $\hat{\gamma}$ & (male) \\
\hline Former Smoker & 수 & 个 & $\boldsymbol{1}$ & \\
\hline Alcohol Ingestion & A & & $<$ & $<$ \\
\hline Diabetes & 숭 & & 4 & $<$ \\
\hline NSAID Usage & $y$ & & & \\
\hline $\begin{array}{l}\text { Menopausal } \\
\text { Hormone Therapy }\end{array}$ & & & & \\
\hline
\end{tabular}

Figure 8: Summation of risk factors for colorectal cancer by anatomical site from Murphy et al. Clin Gastroenterol Hepatol [39]. The doubleheaded arrow means the risk factor is agnostic for that anatomical site (no heterogeneity statistically and no difference between anatomical sites), whereas the downward arrow indicates lowering risk and the upward arrow indicates increasing risk at each anatomic site or overall. CRC, colorectal cancer; NSAID, non-steroidal anti-inflammatory drug. Modified of John M Carethers, Transl Gastroenterol Hepatol [41]. 
Oleg K Glebov et al. [17]: "Distinct epidemiological and clinicopathological characteristics of colorectal carcinomas (CRCs) based on their anatomical location suggest different risk factors and pathways of transformation associated with proximaland distal colon carcinogenesis. These differences may reflect distinct biological characteristics of proximal and distal colonic mucosa, acquired in embryonic or postnatal development, that determine a differential response to uniformly distributed environmental factors. Alternatively, the differences in the epidemiology of proximal and distal CRCs could result from the presence of different procarcinogenic factors in the ascending versus descending colon, acting on cells with either similar or distinct biological characteristics. We applied cDNA microarray technology to explore the possibility that mucosal epithelium from adult proximal and distal colon can be distinguished by their pattern of gene expression. In addition, gene expression was studied infetal (1724 weeks gestation) proximal and distal colon. More than 1000 genes were expressed differentially in adult ascending versus descending colon, with 165 genes showing >2-fold and 49 genes showing $>3$-folddifferences in expression. With almost complete concordance, biopsies of adult colonic epithelium can be correctly classified as proximal or distal by gene expression profile. Only 87 genes were expressed. the differences in incidence of CRC and predilection to microsatellite stable (MSS) or microsatellite unstable (MSI) type of carcinogenesis may: (a) reflect different biological characteristics of proximal and distal colon cells, acquired in embryonic or postnatal development, which in turn determine different responses to common environmental factors; or (b)result from the presence of different procarcinogenic factors in proximal and distal colon (bile acids, bacteria), acting on similar mucosa; or (c) be a combination of a distinct environment and distinct target cells. The incidence of proximal (right) and distal (left) CRCs3 varies depending on the total incidence of CRCs and the geographic location. This indicates that there are different risk factors associated with proximal and distal colon carcinogenesis. Approximately $30 \%$ of all right-sided CRCs are mismatch repair defective as recognized by the presence of an MSI-H, whereas only $2 \%$ of left-sided malignancies manifest the MSI-H phenotype $(3,4)$. This suggests that there may be an inherent or acquired difference between the right and the left colon that makes the right side more susceptible to the initiation or progression of cellular transformation via a pathway that commences with a cell that has lost its ability to recognize and repair nucleotide mismatches. This suggests that there may be an inherent or acquired difference between the right and the left colon that makes the right side more susceptible to the initiation or progression of cellular transformation via a pathway that commences with a cell that has lost its ability to recognize and repair nucleotide mismatches. Relative distinguishing characteristics between right and left-sided CRCs that may reflect the increased number of right-sided MSI-H tumors include several clinicopathological and genetic features. Right-sided CRCs more frequently display undifferentiated and mucinous phenotypes, have pseudo diploid karyotypes, and exhibit a lower frequency of TP53 and K-RAS gene mutations and c-MYC expression. Left-sided tumors, on the other hand, are more uniformly aneuploid with significant loss of heterozygosity (including genes on the 18qchromosome) and rearranged chromosomes and have a higher frequency of TP53 and K-RAS gene mutations and c-MYC expression. The proximal and distal colon have a different embryological origin: cecum, ascending colon, and the proximal two thirds of the transverse colon [proximal, or right colon (RC)] derive from embryonic midgut; the distal third of the transverse colon, descending colon, sigmoid colon and rectum [distal, or left colon (LC)] derive from hindgut [13]. Reflecting their origin, the proximal and distal colon also have a different vascular supply, with the right colon served by the superior mesenteric artery and the left colon by the inferior mesenteric artery. In addition, right colon and left colon differ in expression of several antigens, metabolism of glucose, polyamines and butyric acid, as well as in bile acid concentrations, and composition and density of the bacterial population. Thus, the differences in incidence of CRC and predilection to microsatellite stable (MSS) or microsatellite unstable (MSI)type of carcinogenesis may: (a) reflect different biological characteristics of proximal and distal colon cells, acquired in embryonic or postnatal development, which in turn determine different responses to common environmental factors; or (b)result from the presence of different procarcinogenic factors in proximal and distal colon (bile acids, bacteria), acting on similar mucosa; or (c) be a combination of a distinct environment and distinct target cells. A total of 1349 genes show differences in expression between adult ascending (AT) and descending (L) colon biopsies of patients without colon surgery. Seventy \% (947) of these genes are expressed at a higher level in descending colon and 30\%(402) in ascending colon, suggesting higher transcriptional activity in this overall set of genes in the descending colon.

Comparison with functional gene lists that are present in the Cancer Genome Anatomy Project (CGAP; http://cgap.nci.nih.gov/ in Gene Ontology Browser and Bio Carta Pathways)shows that genes expressed differently in ascending and descending colon are involved in the control of many cellular functions. These include cell cycle, proliferation, cell death, response to external stimuli, stress response, and DNA replication and damage repair. Differentially expressed genes are implicated in major signaling pathways important for colon tumorigenesis, including EGF, TGF, Wnt, Ras, insulin, and integrin signaling. For most of the functional groups, the relative number of genes that are expressed higher in the left compared with the right colon corresponds to a 2:1 ratio that is characteristic of the total set of the CCP genes, but for somegroups the biases are more extreme. For example, $90 \%$ of the genes from cell cycle and DNA metabolism groups are overexpressed in the left colon (see below), whereas the number of overexpressed genes that are involved in cell death, Wnt, and Ras signaling is approximately the same in the ascending and the descending colon". Liu LU et al. [33]: "Propensity to colonic neoplasia differs between the right and left colon. To examine whether this difference may be related to regional differences in epithelial apoptosis, in expression of a proapoptotic regulatory protein, Bak, and in proliferation. 


\section{Individuals with no history of colorectal neoplasia}

Archival blocks of colorectal tissues were immunostained for proliferating cells (antibody to Ki-67 antigen), and Bak expression (polyclonal antiserum). Cells containing DNA strand breaks, a marker of apoptosis, were identified by terminal deoxyuridine nucleotidyl nick end labelling (TUNEL). There were fewer TUNEL positive epithelial cells in the right colon (mean 1.2 (SE 0.1)\% of all epithelial cells) than the left colon $(2.2(0.1) \%$, $\mathrm{p}<0.0001)$ or rectum (2.2 (0.3)\%, p<0.05). Bak expression was less common in the right colon (mean 46 (2.3)\% of epithelial cells immunoreactive) than the left colon (66 (2.7)\%, p<0.0001), or rectum (67 (2.3)\%, p<0.001). Bak expression and TUNEL positivity were highly positively correlated $(\mathrm{p}<0.0001)$. In contrast to apoptosis, mean whole crypt proliferation labelling index was similar throughout the colorectum (right colon: 15.6 (3.2)\%; left colon: 13. 5 (1.2)\%; rectum: 13.3 (2.3)\%). The percentage of proliferating colonic epithelial cells is constant throughout the colon, but fewer epithelial cells undergo Bak mediated apoptosis in the right than in the left colon or rectum. This suggests that colonocytes may be lost by methods other than apoptosis in the right colon ". Qing-Yong Ma et al. [34]: "To investigate the patterns of cell proliferation in proximal and distal colons in normal rats and rats with 1,2-dimethylhydrazine (DMH) induced carcinogenesis using the thymidine analogue bromodeoxyuridine. Colonic crypt cell proliferation was immunohistochemically detected using the anti-bromodeoxyuridine Bu20a monoclonal antibody. Marked regional differences were found in both groups. Total labelling index (LI) and proliferative zone size in both normal $(8.65 \pm 0.34$ vs $7.2 \pm 0.45,27.74 \pm 1.07$ vs $16.75 \pm 1.45)$ and $\mathrm{DMH}$ groups ( $13.13 \pm 0.46$ vs $11.55 \pm 0.45,39.60 \pm 1.32$ vs $35.52 \pm 1.58)$ were significantly higher in distal than in proximal colon $(\mathrm{P}<0.05)$, although the number of cells per proximal crypt was greater $(31.45$ \pm 0.20 vs $34.45 \pm 0.39,42.68 \pm 0.53$ vs $49.09 \pm 0.65, \mathrm{P}<0.0001$ ). Crypt length, total LI and proliferative zone size all increased in both proximal and distal regions of DMH rats compared to normal controls $(\mathrm{P}<0.0001)$. In DMH-treated rat colon a shift of labelled cells to higher crypt cell positions was demonstrated distally whilst a bi-directional shift was evident proximally $(\mathrm{P}<$ 0.05). Our results show that changes in cell proliferation patterns, as assessed by bromodeoxyuridine uptake, can act as a reliable intermediate marker of colonic cancer formation. Observed differences between proliferation patterns in distal and proximal colon may be associated with the higher incidence of tumors in the distal colon". Gertig DM: "Environmental factors, including diet, are important in the etiology of colorectal cancer. Genetic pathways of hereditary and sporadic bowel cancer are being elucidated. Highly penetrant mutations in certain genes, such as mismatch repair genes, play a major role in development of hereditary colorectal cancer, while interactions between low penetrance polymorphisms in carcinogen-metabolizing enzymes and environmental factors may identify susceptible subgroups and shed light on the etiology of sporadic colorectal cancers. In this review we summarize the current state of knowledge regarding the role of gene-environment interactions in development of colorectal cancer and discuss areas of particular promise, as well the limitations of these studies ".

Patricia M Heavey et al. [35]: "Colorectal cancer (CRC) is a significant cause of morbidity and mortality in developed countries, with both genetic and environmental factors contributing to the etiology and progression of the disease. Several risk factors have been identified, including positive family history, red meat intake, smoking, and alcohol intake. Protective factors include vegetables, calcium, hormone replacement therapy, folate, nonsteroidal antiinflammatory drugs, and physical activity. The interaction between these environmental factors, in particular diet and genes, is an area of growing interest. Currently, oncogenes, tumor suppressor genes, and mismatch repair genes are believed to play an essential role in colorectal carcinogenesis. When considering the genetics of CRC, only $10 \%$ of cases are inherited and only $2-6 \%$ can be ascribed to the highly penetrant genes, such as APC, hMLH1 and hMSH2. Lower penetrance genes combined with a Western-style diet contribute to the majority of sporadic CRCs. The purpose of this article is to give a brief overview of the epidemiologic studies that have been conducted and present the major findings. Here, we examine the molecular events in CRC, with particular focus on the interaction between genes and environment and review the most current research in this area". A Povey et al [36]: "The pro-mutagenic lesion 06-methyldeoxyguanosine (O6-MedG), a marker of exposure to many N-nitroso compounds (NOC), can be detected in normal and tumour DNA isolated from colorectal tissue. The biological significance of this exposure is, as yet, unknown but in situ NOC formation is bacterially catalyzed suggesting that NOC formation and potentially DNA alkylation will vary throughout the large bowel.

\section{To determine if 06-MedG levels in colorectal DNA vary within the large bowel}

We studied 62 men and women undergoing surgery for colorectal tumours in the north west of England.

06-MedG levels were measured in paired normal and tumour DNA samples. DNA was digested to nucleosides, fractionated by HPLC, and purified 06-MedG quantified by a radioimmunoassay. 06-MedG was detected in 27 out of a total of 62 (43\%) normal DNA samples and in 30 of 58 (52\%) tumour DNA samples: it was present at concentrations of $<0.01-0.94$ and $<0.01-0.151 \mu \mathrm{mol}$ 06-MedG/mol deoxy guanosine for normal and tumour DNA, respectively. Levels of 06-MedG in normal, but not tumour, DNA from the proximal colon were lower than those found in DNA from either the sigmoid colon $(p=0.03)$ or rectum $(p=0.05)$. When the analysis was restricted to samples that contained 06-MedG, similar results were obtained in that 06-MedG levels in normal DNA were lower in the proximal colon than in the sigmoid colon $(p=0.04)$ or rectum $(p=0.03)$. C ROSSO et al. [37]: "Colorectal cancer (CRC) is an important cause of morbidity and mortality worldwide. A recent systematic analysis for the Global Burden of Disease Study showed that in 2015 there were 1.7 million incident cases of CRC with 832 thousand deaths. According to these data, CRC is considered the third leading cancer and the second leading 
cause of deaths worldwide. Its occurrence is higher in subjects 50 years older dropping by $32 \%$ just since 2000 as the result of screening tools. A lower CRC incidence is registered in South Asia and in Central/Western Sub-Saharan Africa while in Australia, North America and Europe the incidence rate is higher due to the presence of population-based CRC screening. Colorectal cancer originates from the normal epithelial cells of the gastrointestinal tract through a series of sequential genetic (acquired or inherited) and epigenetic alterations. In fact, some individuals are more prone to develop CRC compared to other persons. About $25 \%$ of patients with CRC have a familial background while 15\%show a strong family history that involve a first or a second degree relative. A recent comprehensive integrative analysis of 224 CRC and normal epithelium showed that in more than $90 \%$ of the cases, the majority of the mutations occurred in the genes involved in the WNT signaling pathway, especially in the adenomatous polyposis colon(APC) tumor suppressor gene. The APC protein is ableto interact with the intracellular protein B-catenin that in turn can translocate into the nucleus where stimulates cell proliferation by the activation of c-myc, cyclin D1 and the peroxisome proliferator activated receptor delta (PPARd). B-catenin is involved in cell adhesion making part of the intercellular adhesion complex. Under normal conditions, when B-catenin levels increase, APC protein promotes its apoptosis through the proteasome pathway. If APC is mutated, B-catenin accumulates into the nucleus leading to the formation of adenoma. Most of the patients carrying APC mutations develop benign adenomas but some of them accumulate additional genetic aberrations over time that lead to the inactivation of thep53 gene thus promoting the conversion to the malignant phenotype. Another mechanism of CRC leading to genomic instability can arise from the $\mathrm{CpG}$ island methylator phenotype pathway, a common epigenetic event that occur in cancer. For example, hypomethylation, a decrease in methylation on cytosine that occur mainly in the repetitive DNA sequences, is a characteristic feature observed in hyperplastic and denomatous polyps. mutations in the DNA mismatch repair genes that are involved in the correction of DNA errors after the replication process(AT Pases hMSH2, hMSH6, hMSH3, hMLH1, hPMS2,hPMS1 and hMLH3 genes), may lead to microsatellite (short tandem repeat) instability that is found in about $15 \%$ of CRC.

DNA alkylation varied within the large bowel possibly due to in situ NOC formation and was highest in areas of the colon and rectum where the highest incidence of large bowel the aim of the screening is to reduce both incidence and mortality of CRC. The effectiveness of screening programs depends on the adherence of the eligible population and on the sensitivity of the screening methods proposed. Colonoscopy, FS and FIT, are the main screening tests proposed for the identification of precancerous lesions or CRC but there is also a growing interest in the implementation of other noninvasive tests, particularly those based on stool. To date, the participation rate to the screening programs remains low and more strategies should be proposed to improve population adherence. About $20 \%$ of subjects who adhere for the first time to a screening program, do not adhere to a second invitation. This aspect should be analyzed carefully because it represents an important limitation of the screening. Future studies should clarify the potential role of miRNAs as noninvasive markers of CRC. Finally, fascinating fields of research as that regarding the potential involvement of molecules as ADAM (A disintegrin and metalloprotease) in carcinogenesis and consequently their potential utilization as biomarkers need deep studies before the translation of laboratory information in clinical practice". "Another important trigger for the development of CRC is chronic inflammation as documented by the strong association between inflammatory bowel diseases (IBD) and CRC. Inflammation favors the activation of the antiapoptotic system increasing DNA damage through the activation of reactive oxygen species. Chronic elevated levels of tumor necrosis factor alpha (TNF-a)promote tumor growth, proliferation and metastasis whileinterleukin-6 (IL-6) stimulates the transcription of STAT 3favoring its translocation into the nucleus and promoting cell proliferation and differentiation through the activation of some genes transcription such as vascular endothelial growth factor receptor 2 (VEGFR2), Bcl-2, Cyclin D1,MMP2-9, ICAM-1, and COX2. GP Caviglia et al [38]: "Colorectal cancer (CRC) is the third most frequent cancer in humans and is a major health problem in developed countries. More than $90 \%$ of cases originate from colorectal adenoma following a long, stepwise carcinogenic process. Thus, the early detection and removal of adenomatous lesions are crucial in preventing mortality and morbidity due to CRC. Currently, fecal immunochemical test for hemoglobin (FIT) is considered the standard approach for population-screening programs whereas total colonoscopy represents the gold standard for CRC diagnosis. Both FIT and colonoscopy have several limitations including low sensitivity and specificity for the former, and low acceptance rate and expensiveness for the latter. Consequently, there is fervent interest towards finding new strategies and new approaches for CRC screening “.

L Thomas et al. [39]: "Deoxycholic acid (DCA), implicated in the pathogenesis of gall stones and colorectal cancer, is mainly formed by bacterial deconjugation (cholylglycine hydrolase (CGH)) and $7 \alpha$-dehydroxylation ( $7 \alpha$-dehydroxylase $(7 \alpha-\mathrm{DH})$ ) of conjugated cholic acid (CA) in the caecum/proximal colon. most previous studies of CGH and $7 \alpha$-DH have been in faeces rather than in caecal contents. In bacteria, CA increases $7 \alpha$-DH activity by substrateenzyme induction but little is known about CA concentrations or $\mathrm{CA} / 7 \alpha-\mathrm{DH}$ induction in the human colon. In fresh "faeces", and in caecal aspirates obtained during colonoscopy from 20 patients, we: (i) compared the activities of CGH and $7 \alpha-\mathrm{DH}$, (ii) measured $7 \alpha-$ DH in patients with "low" and "high" percentages of DCA in fasting serum (less than and greater than the median), (iii) studied CA concentrations in the right and left halves of the colon, and examined the relationships between (iv) $7 \alpha$-DH activity and CA concentration in caecal samples (evidence of substrate-enzyme induction), and (v) $7 \alpha-\mathrm{DH}$ and per cent DCA in serum. Although mean CGH activity in the proximal colon $(18.3$ (SEM 4.40) $\times 10-2 \mathrm{U} / \mathrm{mg}$ protein) was comparable with that in "faeces" $(16.0(4.10) \times 10-2 \mathrm{U} / \mathrm{mg}$ protein $)$ 
, mean $7 \alpha$-DH in the caecum $(8.54$ (1.08) $\times 10-4 \mathrm{U} / \mathrm{mg}$ protein) was higher $(\mathrm{p}<0.05)$ than that in the left colon $(5.72(0.85) \times 10-4 \mathrm{U} / \mathrm{mg}$ protein). At both sites, $7 \alpha$-DH was significantly greater in the "high" than in the "low" serum DCA subgroups. CA concentrations in the right colon $(0.94(0.08) \mu \mathrm{mol} / \mathrm{ml})$ were higher than those in the left $(0.09(0.03) \mu \mathrm{mol} / \mathrm{ml} ; \mathrm{p}<0.001)$ while in the caecum (but not in the faeces) there was a weak $(\mathrm{r}=0.58)$ but significant $(\mathrm{p}<0.005)$ linear relationship between $7 \alpha-\mathrm{DH}$ and CA concentration. At both sites, $7 \alpha$-DH was linearly related $(\mathrm{p}<0.005)$ to per cent DCA in serum. (i) confirm that there are marked regional differences in bile acid metabolism between the right and left halves of the colon, (ii) suggest that caecal and faecal $7 \alpha$-DH influence per cent DCA in serum (and, by inference, in bile), and (iii) show that the substrate CA induces the enzyme $7 \alpha-\mathrm{DH}$ in the caecum".

Cassidy A et al. [40]: "Intakes of starch, non-starch polysaccharides (NSPs), protein and fat have been compared with colorectal cancer incidence in 12 populations worldwide. There were strong inverse associations between starch consumption and large bowel cancer incidence (large bowel $r=-0.70$, colon $r=$ -0.76).There was no significant relation with NSPs, although the association with large bowel cancer incidence was still significant when NSP was combined with resistant starch (RS) to give an estimate of fermentable carbohydrate (large bowel $r=-0.52$, colon $r=-0.60$ ). The relationships between starch, RS and NSPs and cancer incidence remained statistically significant after adjusting for fat and protein intakes. The strong inverse associations found here suggest a potentially important role for starch in protection against colorectal cancer and correspond with the hypothesis that fermentation in the colon is the mechanism for preventing colorectal cancer. Measures of both starch and NSPs need to be included in future epidemiological studies of diet and bowel cancer". John M. Carethers [41]: Risk factors for colon location of cancer, Transl Gastroenterol Hepatol "World-wide, humans developed 1,096,601 colon and 704,376 rectal cancers during 2018, accounting for $10 \%$ of all new cases of cancer (1). Additionally, 551,269 people died of colon cancer and another 310,394 people died of rectal cancer, accounting for $9 \%$ of total cancer deaths (1). The disease is most prevalent in Western countries (1). In the US from 2009 to 2013, the distribution of cancers to the lower gastrointestinal tract was $41 \%$ in the proximal colon (proximal and including the splenic flexure), $22 \%$ in the distal colon (descending and sigmoid), $28 \%$ in the rectum, and $8 \%$ other sites (2). This anatomical site distribution slightly varied by gender, with females having $46 \%, 21 \%, 25 \%$, and $8 \%$ for proximal, distal, rectum and other, respectively, and males having a 37\%, 24\%, 32\%, and $7 \%$ distribution (2). There are a number of hypotheses that attempt to explain the varied distribution including why females develop more proximal cancers than men (3). These include the different embryological origins of the proximal and distal colons, variations in the concentration of bile salts and other compounds, level of oxygenation, and the microbial environment in different locations within the colon (3). Women overall live longer than men, and there is a distal-to-proximal shift of colon cancer as we age (4). The influence of sex hormones is also implicated (3). There are several morphologic and genetic observations between proximal and distal colon cancers that highlight the differences in colon location (57). Morphologically, flat sessile serrated adenomas and cancers are more commonly found in the proximal colon, and are difficult to observe without enhanced colonoscopic techniques, whereas polypoid adenomas and cancers are more common in the distal colon (3,5-7). Genetically, hypermethylation of the DNA mismatch repair gene MLH1 causing microsatellite instability is much more likely in proximal cancers, and tumors are diploid and generally lack TP53 mutations. Additionally, these sporadic microsatellite unstable cancers are also associated with the V600E mutation of BRAF (6). Chromosomal unstable tumors are more common in the distal colon, are aneuploid, and are driven by biallelic inactivation of APC and TP53 genes " [42-53].

\section{Experimental project hypothesis}

In order to verify the hypothesis that 2 different kind of " toxicpathological" process are involved in the different form RIGHT and LEFT of colon cancer is possible to cross the epidemiological data related kind of diet (advanced countries vs non advanced), north vs south of the world, and the prevalence of the 2 form of CRC. Diet is a modifiable factor (that is considered as a moderate toxic or preventing - protective). But in physiopathology of colon disease there are gradients in some factors (biliar salts , microbioma, Water intake physiology, concentration of waste materials and so on). A different prevalence in $\mathrm{L}-\mathrm{R}$ CRC verified in different environment is related to modifiable factor and not due to A mutation.( not only a pure stochastic event).

\section{Discussion}

From literature analysis is possible to verify that colon cancer presents some relevant differences in left vs right form. "Left-sided CRC predominantly follow the CIN molecular pathway. Right-sided CRC predominantly characterized by microsatellite instability and are hypermutated tumors"[12]. These 2 forms are related or to an MSI or to CIN p-53 KRAS profile. This is an interesting fact if it is considered that the same kind of tissue (large bowel) produce 2 different kind of cancer topographic related (left vs right).

It is also interesting to verify some local condition and the presence of a kind of different 2 kinds "toxic gradient factors" acting in the 2 different topographic bowel region (left and right):in example Due by "Anatomically, the content and variety of microbiota differs from proximal and distal colorectum. Fermentation reactions produce short-chain fatty acids at significantly higher levels in the distal colorectum compared to the proximal colon. The higher bacterial load in the distal colorectum results in more hydrolytic and reductive bacterial enzymes involved in production of mutagenic metabolites than found more proximally" [14]. Other gradient factor can be biliary salt concentration. "Recent studies demonstrate that patients with advanced right-sided colon cancers have a worse prognosis than those with left-sided colon or rectal cancers, and these patient subgroups respond differently to biological therapies "[27]. IBD and history of CRC in first-degree 
relatives are associated with much higher risk of CRC. Increased BMI, red meat intake, cigarette smoking, low physical activity, low vegetable consumption, and low fruit consumption were associated with moderately increased risk of CRC" (Table 2).

Table 2: Relative Risks for Established Colorectal Cancer Risk Factors [43].

\begin{tabular}{|c|c|}
\hline Factors that Increase Risk & Relative Risk* \\
\hline \multicolumn{2}{|l|}{ Heredity and medical history } \\
\hline \multicolumn{2}{|l|}{ Family history } \\
\hline 1first-degree relative 46 & 2.2 \\
\hline More than 1 relative 46 & 4 \\
\hline Relative with diagnosis before age 4554 & 3.9 \\
\hline Inflammator y bowel disease47 & 1.7 \\
\hline Diabetes48 & 1.3 \\
\hline \multicolumn{2}{|l|}{ Behavioral Factors } \\
\hline \multicolumn{2}{|l|}{ Alcohol consumption (daily average) } \\
\hline 2-3 drinks & 1.2 \\
\hline$>3$ drinks 1.4 & 1.3 \\
\hline Obesity (body mass index $\geq 30 \mathrm{~kg} / \mathrm{m} 2$ ) 49 & 1.2 \\
\hline Red meat consumption $(100 \mathrm{~g} /$ day $) 50$ & 1.2 \\
\hline Processed meat consumption $(50 \mathrm{~g} /$ day $) 50$ & 1.2 \\
\hline \multicolumn{2}{|l|}{ Smoking (ever vs. never)51 } \\
\hline \multicolumn{2}{|l|}{ Factors that Decrease Risk: } \\
\hline Physical activity (colon) 52 & 0.7 \\
\hline Dair y consumption ( $400 \mathrm{~g} /$ day $) 53$ & 0.8 \\
\hline Milk consumption $(200 \mathrm{~g} /$ day $) 53$ & 0.7 \\
\hline $\begin{array}{l}\text { *Relative risk compares the risk of disease among } \\
\text { people with a particular "exposure" to the risk among } \\
\text { people without that exposure. Relative risk for } \\
\text { dietary factors compares the highest with the lowest } \\
\text { consumption. If the relative risk is more than 1.0, } \\
\text { then risk is higher among exposed than unexposed } \\
\text { persons. Relative risks less than } 1.0 \text { indicate a } \\
\text { protective effect }\end{array}$ & \\
\hline
\end{tabular}

\section{Conclusion}

As in analytical chemical field often is used a so called "internum standard" to better study concentration of a substantia in a medium the same the 2 form of colon cancer left, and right can be a useful instrument to better understand 2 different kind of neoplasia in the same tissue. Is possible to verify epidemiology of colon cancer and IBS and verify that diet is very different in advanced from other countries. Two different kind of neoplasia in 2 different bowel zone seem due to 2 different gradients of a kind of "toxic " movens or pathological process (In right and left bowel part). Many differences are present if proximal vs distal colon and this support a different kind of local environment. This model can be useful to explain in a different way the carcinogenesis process for further research. A final consideration: if are considered the global cost due by meat diet in advanced countriesCO2 production, cost of transport, cost of diagnostic procedure, cost of drugs for colon cancer is a real paradox. This socio-economic factor is involved in availability of mass screening test but also in kind of diet.

\section{Clarification}

This article is produced under a pharmacological and pharmaceutical point of view, without any therapeutic or diagnostic intent only to produce new research hypothesis to be submitted to the researcher.

\section{Acknowledgment}

None.

\section{Conflict of Interest}

No conflict of interest.

\section{References}

1. Peters U, Bien S, Zubair N (2015) Genetic Architecture of Colorectal Cancer. Gut 64(10):1623-1636.

2. Carethers JM (2011) One Colon Lumen but Two Organs. Gastroenterology 141(2): 411-412.

3. Hosein R, Reza P, Mehdi A, Yones K, Jalal M, et al. (2016) Colorectal cancer in Iran: Epidemiology and morphology trends. EXCLI J 15:738-744.

4. Johnson CM, Wei C, Ensor JE, Smolenski DJ, Amos CI, et al. (2013) Meta-analyses of colorectal cancer risk factors. Cancer Causes Control 24(6):1207-1222.

5. Vogelstein B, Fearon ER, Hamilton SR, Kern SE, Preisinger AC, et al. (1988) Genetic alterations during colorectal-tumor development. N Engl J Med 319(9): 525-532.

6. Burcin Barana, Nazli Mert Ozupeka, Nihal Yerli Tetika, Emine Acarb, Omer Bekcioglu (2018) Difference Between Left-Sided and Right-Sided Colorectal Cancer: A Focused Review of Literature. Gastroenterol Res 11(4): 264-273.

7. Söreide K", Janssen EAM, Iland H So", Korner H, Baak JPA (2001) Microsatellite instability in colorectal cancer. American Journal of Pathology 158(4).

8. Laura AE Hughes, Colinda CJM Simons, Piet A van den Brandt, Manon van Engeland, Matty P Weijenberg (2017) Lifestyle, Diet, and Colorectal Cancer Risk According to (Epi)genetic Instability: Current Evidence and Future Directions of Molecular Pathological Epidemiology. Curr Colorectal Cancer Rep 13(6): 455-469.

9. Inés M, Sánchez-de-Diego C, Pradilla Dieste A, Cerrada E, Rodriguez Yoldi MJ (2017) Colorectal Carcinoma: A General Overview and Future Perspectives in Colorectal Cancer. Int J Mol Sci 18(1)- E197.

10. Nagao-Kitamoto H, Kitamoto S, Kuffa P, Kamada N (2016) Pathogenic role of the gut microbiota in gastrointestinal diseases. Review Intestinal Res 14(2): 127-138.

11. Ha Thi Nguyen, Hong-Quan Duong (2018) The molecular characteristics of colorectal cancer: Implications for diagnosis and therapy. Oncology Letters 16: 9-18.

12. Camille Thélin, Sanjay Sikka (2015) Epidemiology of Colorectal CancerIncidence, Lifetime Risk Factors Statistics and Temporal Trends.

13. Lee GH, Malietzis G, Askari A, Bernardo D, Al-Hassi HO, et al. (2015) Is right-sided colon cancer different to left-sided colorectal cancer? Eur J Surg Oncol 41(3): 300-308.

14. McBain AJ, Macfarlane GT (1998) Ecological and physiological studies on large intestinal bacteria in relation to production of hydrolytic and reductive enzymes involved in formation of genotoxic metabolites. J Med Microbiol 47(5): 407-416.

15. Povey AC, Hall CN, Badawi AF, Cooper DP, O'Connor PJ (2000) Elevated levels of the pro-carcinogenic adduct, $\mathrm{O}(6)$-methylguanine, in normal DNA from the cancer prone regions of the large bowel. Gut 47(3): 362365.

16. Larsson SC, Rafter J, Holmberg L, Bergkvist L, Wolk A (2005) Red meat consumption and risk of cancers of the proximal colon, distal colon and 
rectum: the Swedish Mammography Cohort. Int J Cancer 113(5): 829834.

17. Glebov OK, Rodriguez LM, Nakahara K, Jenkins J, Cliatt J, et al. (2003) Distinguishing right from left colon by the pattern of gene expression. Cancer Epidemiol Biomarkers Prev 12(8): 755-762.

18. Hachichmid P, Nasr K, Mohallatmede EA, Ghassemmid H, Sadrim S, et al. (1977) Colorectal polyps and carcinoma in Southern Iran. Cancer 39: 274-278.

19. Harpaz N, Polydorides AD (2010) Colorectal Dysplasia in Chronic Inflammatory Bowel Disease Pathology, Clinical Implications, and Pathogenesis. Arch Pathol Lab Med 134(6): 876-895.

20. Schneider A, Stolte M (1993) Clinical and pathomorphological findings in patients with colorectal carcinoma complicating ulcerative colitis. Z Gastroenterol 31(3): 192-197.

21. Riegler G, Bossa F, Caserta L, Pera A, Tonelli F, et al. (2003) IG-IBD Group. Colorectal cancer and high-grade dysplasia complicating ulcerative colitis in Italy. Dig Liver Dis 35(9): 628-634.

22. Pasquereau-Kotula E, Mariana M, Laetitia A (2018) Significance of Streptococcus gallolyticus subsp. gallolyticus Association with Colorectal Cancer. Front Microbiol 9: 614

23. Ritesh K, Jennifer LH, Deborah S, Jennifer D, Scott K, et al. (2017) Streptococcus gallolyticus subsp. gallolyticus promotes colorectal tumor development. PLoS Pathog 13(7): e1006440.

24. Macfarlane GT, Gibson GR, Cummings JH (1992) Comparison of fermentation reactions in different regions of the human colon . J Appl Bacteriol 72(1): 57-64.

25. Jianhong P, Cong L, Fulong W, Huizhong Z, Weiwei X, et al. (2018) Right- and left-sided stage III colon cancers present different prognostic outcomes of oxaliplatin-based adjuvant chemotherapy after curative resection. Cancer Manag Res 10: 2095-2103.

26. Salem ME, Weinberg BA, Xiu J, El-Deiry WS, Hwang JJ, et al. (2017) Comparative molecular analyses of left-sided colon, right-sided colon, and rectal cancers. Oncotarget 8(49): 86356-86368.

27. Lim DR, Kuk JK, Kim T, Shin EJ (2017) Comparison of oncological outcomes of right-sided colon cancer versus left-sided colon cancer after curative resection .Which side is better outcome? Medicine (Baltimore) 96(42): e8241.

28. Qiu MZ, Pan WT, Lin JZ, Wang ZX, Pan ZZ, et al. (2018) Comparison of survival between right-sided and left-sided colon cancer in different situations . Cancer Med 7(4): 1141-1150.

29. Wang Y, Yang L, Zhou M, Shen L, Zhang J, et al. (2018) Disparities in survival for right-sided vs. left-sided colon cancers in young patients: a study based on the Surveillance, Epidemiology, and End Results database (1990-2014).Cancer Manag Res 10: 1735-1747.

30. Tiratterra E, Franco P, Porru E, Katsanos KH, Christodoulou DK, et al. (2018) Role of bile acids in inflammatory bowel disease. Ann Gastroenterol 31(3): 266-272.

31. Aykan NF (2015) Red Meat and Colorectal Cancer . Oncol Rev 9(1): 288.

32. Norat T, Bingham S, Ferrari P, Slimani N, Jenab M, et al. (2005) Meat, fish, and colorectal cancer risk: the European Prospective Investigation into cancer and nutrition .J Natl Cancer Inst 97(12): 906-916.

33. Liu LU, Holt PR, Krivosheyev V, Moss SF (1999) Human right and left colon differ in epithelial cell apoptosis and in expression of Bak, a proapoptotic Bcl-2 homologue. Gut 45(1): 45-50.

34. Ma QY, Williamson KE, Rowlands BJ (1998) Variability of cell proliferation in the proximal and distal colon of normal rats and rats with dimethylhydrazine induced carcinogenesis. Semin Cancer Biol 8(4): 285-298.
35. Heavey PM, McKenna D, Rowland IR (2004) Colorectal Cancer and the Relationship Between Genes and the Environment. Nutr Cancer 48(2):124-141.

36. Povey A, Hall C, Badawi A, Cooper D, Connor P (2000) Elevated levels of the pro-carcinogenic adduct, 06-methylguanine, in normal DNA from the cancer prone regions of the large bowel. Gut 47(3): 362-365.

37. Chiara Rosso, Luca Cabianca, Fabrizio M Gili (2019) Non-invasive markers to detect colorectal cancer in asymptomatic population. Minerva Biotecnologica 31(1):23-29.

38. Caviglia GP, Cabianca L, Fagoonee S, Gili FM (2016) Colorectal cancer detection in an asymptomatic population: fecal immunochemical test for hemoglobin vs. fecal M2-type pyruvate kinase. Biochemia Medica 26(1): 114-120.

39. Thomas LA, Veysey MJ, French G, Hylemon PB, Murphy GM (2001) Bile acid metabolism by fresh human colonic contents: a comparison of caecal versus faecal samples. 49(6): 835-842.

40. Cassidy A, Bingham SA, Cummings JH (1994) Starch intake and colorectal cancer risk: an international comparison. Br J Cancer 69(5): 937-942.

41. Carethers JM (2018) Risk factors for colon location of cancer. Transl Gastroenterol Hepatol 3: 76.

42. American Cancer Society (2017) Colorectal Cancer Facts \& Figures 2017-2019. Atlanta, USA.

43. Butterworth AS, Higgins JP, Pharoah P (2006) Relative and absolute risk of colorectal cancer for individuals with a family history: a metaanalysis. Eur J Cancer 42: 216-227.

44. Lutgens MW, van Oijen MG, van der Heijden GJ, Vleggaar FP, Siersema PD, et al. (2013) Declining risk of colorectal cancer in inflammatory bowel disease: an updated meta-analysis of population-based cohort studies. Inflamm Bowel Dis 19: 789-799.

45. Tsilidis KK, Kasimis JC, Lopez DS, Ntzani EE, Ioannidis JP (2015) Type 2 diabetes and cancer: umbrella review of meta-analyses of observational studies. BMJ 350: g7607.

46. Ma Y, Yang Y, Wang F, Zhang P, Shi C, et al. (2013) Obesity and risk of colorectal cancer: a systematic review of prospective studies. PLoS One 8(1): e53916136.

47. Chan DS, Lau R, Aune D, Vieira R, Greenwood DC, et al. (2011) Red and processed meat and colorectal cancer incidence: meta-analysis of prospective studies. PLoS One. 6(6): e20456145.

48. Botteri E, Iodice S, Bagnardi V, Raimondi S, Lowenfels AB, et al. (2008) Smoking and colorectal cancer: a meta-analysis. Jama 300: 2765-2778.

49. Boyle T, Keegel T, Bull F, Heyworth J, Fritschi L (2012) Physical activity and risks of proximal and distal colon cancers: a systematic review and meta-analysis. J Natl Cancer Inst 104: 1548-1561.

50. Aune D, Lau R, Chan DS, Vieira R, Greenwood DC, et al. (2012) Dairy products and colorectal cancer risk: a systematic review and metaanalysis of cohort studies. Ann Oncol 23: 37-45.

51. Johns LE, Houlston RS (2001) A systematic review and meta-analysis of familial colorectal cancer risk. Am J Gastroenterol 96(10): 2992-3003.

52. Canavan C, West J, Card T (2014) The epidemiology of irritable bowel syndrome. Clinical epidemiology 6: 71-80.

53. Schutte AE (2017) Global, regional, and national age-sex specific mortality for 264 causes of death, 1980-2016: a systematic analysis for the Global Burden of Disease Study 2016. Lancet 390(10100):11511210.

Citation: Mauro Luisetto, Behzad Nili Ahmadabadi, Hossein Nili-Ahmadabadi, Ahmed Yesvi Rafa, Gamal Abdul Hamid, et al. Comparison of Risk Factors and Molecular Analysis of Right-Sided Colon and Left Sided Colon Cancer. Adv Can Res \& Clinical Imag. 2(2): 2019. ACRCI 